\title{
Shallow water model on cubed-sphere by multi-moment finite volume method
}

\author{
Chungang Chen ${ }^{\mathrm{a}}$, Feng Xiao ${ }^{\mathrm{a}, \mathrm{b}, *}$ \\ a Department of Energy Sciences, Tokyo Institute of Technology, 4259 Nagatsuta, Midori-ku, Yokohama 226-8502, Japan \\ ${ }^{\mathrm{b}}$ DES/LHD, Institute of Mechanics, Chinese Academy of Sciences, 15 Beisihuanxi Road, Beijing 100080, China
}

Received 10 May 2007; received in revised form 12 January 2008; accepted 17 January 2008

Available online 2 February 2008

\begin{abstract}
A global numerical model for shallow water flows on the cubed-sphere grid is proposed in this paper. The model is constructed by using the constrained interpolation profile/multi-moment finite volume method (CIP/MM FVM). Two kinds of moments, i.e. the point value (PV) and the volume-integrated average (VIA) are defined and independently updated in the present model by different numerical formulations. The Lax-Friedrichs upwind splitting is used to update the PV moment in terms of a derivative Riemann problem, and a finite volume formulation derived by integrating the governing equations over each mesh element is used to predict the VIA moment. The cubed-sphere grid is applied to get around the polar singularity and to obtain uniform grid spacing for a spherical geometry. Highly localized reconstruction in CIP/MM FVM is well suited for the cubed-sphere grid, especially in dealing with the discontinuity in the coordinates between different patches. The mass conservation is completely achieved over the whole globe. The numerical model has been verified by Williamson's standard test set for shallow water equation model on sphere. The results reveal that the present model is competitive to most existing ones.
\end{abstract}

(c) 2008 Elsevier Inc. All rights reserved.

Keywords: Finite volume method; Cubed-sphere grid; Shallow water equations; Spherical geometry; Global model; High-order scheme; Geophysical flows

\section{Introduction}

How to represent the spherical geometry is one of the key points to the numerical simulations on the large scale circulation of the Earth's atmosphere. In the context of finite difference formulation, latitudelongitude (LAT/LON) grid has gained a greater popularity compared to other grids for spherical geometry, such as the icosahedron geodesic grid [30,35] and the cubed-sphere grid [29] which were proposed more than 30 years ago though. The standard LAT/LON grid, however, meets substantial difficulties in regions close

\footnotetext{
* Corresponding author. Address: DES/LHD, Institute of Mechanics, Chinese Academy of Sciences, 15 Beisihuanxi Road, Beijing 100080, China. Tel./fax: +861082543877.

E-mail address: xiao@imech.ac.cn (F. Xiao).
} 
to the poles, i.e. the singularities in the governing equations and the convergence of meridians. Although the numerical barriers in the LAT/LON grid can be circumvented to some extent by using special numerical techniques, for example the polar cap [9], the semi-Lagrangian/semi-implicit formulation [5] and the reduced LAT/LON grid [26], the large ratio in the grid spacing seriously prevents the uniformly resolved numerical solutions on the whole sphere. Moreover, these problems are getting worse as the spatial resolution is increasingly refined.

Other grids with more uniform resolution for the whole globe began to draw more attention since the middle of 1990's, an age from which the GCM simulations with high resolution became technically possible due to the rapid development of computer hardware. Two representatives of the grids for spherical geometry, i.e. the gnomonic or conformal cubic grid $[18,19,25,27,28]$ and the icosahedron geodesic grid $[8,16,31,34]$ have been adopted in global models for either shallow water models or atmospheric models. The cubed-sphere grid is generated by mapping the sphere onto an inscribed cube using the gnomonic projection. A expanded spherical cube is composed of six identical patches connected to each other by 12 edges which are also called patch boundaries. A gnomonic projection results in a more uniform grid spacing, but the mesh on each patch is not orthogonal. Furthermore, the coordinate across a patch boundary is not continuous. As commentated in [25], the "breaking" of the coordinate along the patch boundaries requires extra numerical treatments that usually degrade the accuracy of numerical solution. A remedy to the non-orthogonality of the gnomonic cubic grid is the conformal cubic grid [25]. McGregor [18] implemented the semi-Lagrangian method on the conformal cubic grid and constructed a global atmospheric model with some special numerical technique [20].

More accurate numerical formulations on the cubed-sphere grid have been recently devised by implementing some advanced numerical methods, i.e. the spectral element method [7,32] and the discontinuous Galerkin (DG) method [23,24]. The DG method [4] computes the volume-integrated value over each control volume (mesh cell) via a finite volume formulation, which then guarantees the numerical conservation. High order reconstruction can be built locally in a DG method by increasing the local degrees of freedom. The DG method, however, involves numerical quadrature which is computationally expensive, and moreover, the CFL condition for a high order DG method is much restrictive as discussed in [44].

We have recently developed another type of high resolution scheme, namely constrained interpolation profile/multi-moment finite volume method (CIP/MM FVM) for fluid dynamic simulations. Different from the conventional finite volume methods, the CIP/MM FVM employs at least two kinds of quantities which are generically called "moment" in our context, e.g. the point value (PV) and the volume-integrated averages (VIA) of a field variable, as the prognostic variables. The moments are put forward in time separately using different numerical algorithms. For example, the PV is updated by solving a point-wise Riemann problem or a semi-Lagrangian procedure, while the VIA has to be computed via a finite volume scheme of flux-form to assure the conservation. A CIP/MM FVM allows larger CFL number for stability and is more computationally efficient. The interpolation reconstruction, which is required for the computations of the Riemann problem and the numerical flux, is built in terms of both PV and VIA, thus the mesh stencil used in the reconstruction is very compact. Using multi-moments as the model variables in constructing FVM has been implemented to CFD problems so far in [10,11,37,38]. The multi-moment formulation may result in numerical dispersion property different from the conventional finite difference methods. In [39], for example, the numerical dispersion of the simplest multi-moment finite volume method for the geostrophic adjustment was discussed. In [10], a high order advection scheme was developed for unstructured grid where a cubic interpolation function is constructed over a single triangular mesh by using both PV and VIA moments. The local reconstruction makes the CIP/MM FVM well suited not only for the unstructured grid but also for the numerical treatment of the patch boundary in the cubed-sphere grid.

We present in this paper a CIP/multi-moment finite volume scheme for shallow water equation model on cubed-sphere grid with equiangular gnomonic projection. The PV moment is defined at the vertices and the middle points of the boundary edges, while the VIA is defined over the $2 \mathrm{D}$ quadrilateral control volume for each mesh element. Only one layer of ghost cells are required across the patch boundaries during updating $\mathrm{PV}$. The computation over the patch boundaries of the gnomonic cube can be carried out by mapping the ghost cells from the neighboring patch. 
This paper is organized in the following way. In Section 2, a brief introduction to the gnomonic cubed grid and the governing equations developed for curvilinear coordinates are given. Then a detailed description of our multi-moment numerical model for solving two-dimensional shallow water flow on a curvilinear grid and the numerical treatments of the patch boundary are presented in Section 3. Several numerical tests on the sphere are carried out in Section 4 to verify the present scheme. Finally, some conclusions are given in Section 5.

\section{The cubed-sphere grid}

\subsection{Cubed-sphere geometry}

On a cubed-sphere, the sphere surface is divided into six identical patches by projecting the six rectangular sides of an inscribed cube onto the sphere surface, shown in Fig. 1. For every patch, identical local coordinates can be constructed. The governing equations written in the local coordinates have the same appearance for all patches. A cubed-sphere grid can get around the polar singularities in the LAT/LON grid, and is able to generate very uniform grid spacing over the whole globe.

Two kinds of projections are often adopted to construct the local coordinates on cubic-sphere, i.e. the gnomonic projection originally proposed by Sadourny [29] and used in $[19,23,34,25,27,28]$ and the conformal projection proposed by Rancic [25] and used in [18,2]. The gnomonic projection produces a more uniform grid on the sphere, but encounters the discontinuity of the coordinates at the boundary edges between the different patches. Moreover, the coordinate system in a gnomonic projection is not orthogonal and the governing equations appear to be more complicated than in an orthogonal coordinate system. The conformal projection, on the other hand, gives a continuous patch connection and a simpler form of the governing equations. However, a conformal projection results in a converged grid lines around the eight vertices of the inscribed cube and there are no analytic metric terms for conformal projection. Recently, the uniformity of conformal cubic grid is improved by Adcroft et al. [see [2], Fig. 7].

We adopt the gnomonic projection in present study. In order to minimize boundary regions among different patches and to construct the interpolation required for exchanging data more efficiently, two kinds of moments are used and the reconstruction is carried out on a local base. The resultant numerical algorithm is more convenient to deal with the boundaries with broken coordinates and maintain the numerical conservation.

In a gnomonic equiangular projection, the local grid on each patch is constructed by intersection of two sets of angularly equidistant great circles, see their Fig. 1 for patch one in [27]. The location of any point on patch one can be determined by central angles $(\alpha, \beta)$ which are the angles between the great circles passing this point and the great circles passing the center of the patch in $\xi$ and $\eta$ directions respectively. This procedure can be repeated six times to produce the grids on six patches covering the whole globe. The central

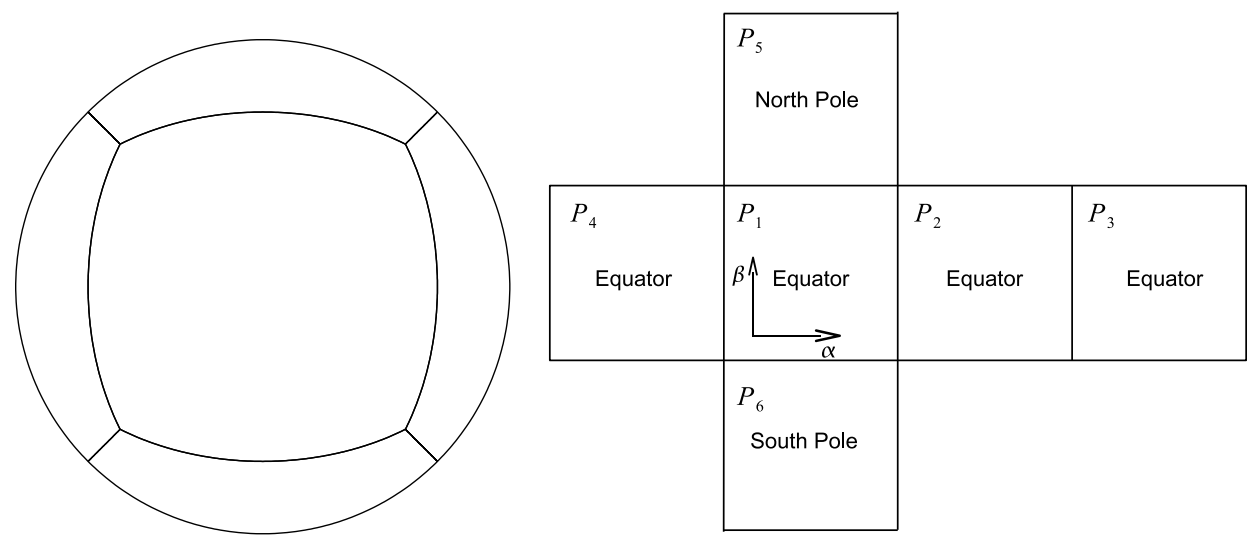

Fig. 1. The cubed-sphere. 
angles $(\alpha, \beta)$ vary in $\left[-\frac{\pi}{4}, \frac{\pi}{4}\right]$ for every patch. In our study the local coordinates $(\xi, \eta)$ are defined as length of the arcs, $\xi=R \alpha$ and $\eta=R \beta$, instead of the original angular variables where $R=6.371220 \times 10^{6} \mathrm{~m}$ is the radius of the Earth.

The projection relations of patch one are shown in Fig. 2. $P$ is a point on the sphere surface and $P^{\prime}$ is the corresponding point on the cube's surface. The transformation laws between spherical LAT/LON coordinates $(\lambda, \theta)$, where $\lambda$ is the longitude and $\theta$ is the latitude, and the local coordinates $(\xi, \eta)$ for patch one can be deduced from the geometrical relations as

$$
\left\{\begin{array}{l}
\xi=R \lambda, \\
\eta=R \arctan \left(\tan \theta \cos ^{-1} \lambda\right)
\end{array}\right.
$$

The transformation laws (1) are satisfied for any patch in rotated LAT/LON coordinates with the origin located at the center of the corresponding patch. With the application of the transformation formulations between the rotated and the original LAT/LON coordinates [17], the transformation laws for any other patch can be obtained. The detail expressions can be found in ([24], Appendix A).

In a non-orthogonal grid, two sets of base vectors are needed to describe a vector field, which are the covariant vector and contravariant vector, the corresponding velocities are covariant velocity $(u, v)$ and the contravariant velocity $(\tilde{u}, \tilde{v})$. Given the covariant (tangent) base vector as $\mathbf{a}_{1}$ and $\mathbf{a}_{2}$, the covariant velocity vector is defined as $u=\mathbf{v} \cdot \mathbf{a}_{1}$ and $v=\mathbf{v} \cdot \mathbf{a}_{2}$, the contravariant velocity satisfies $\mathbf{v}=\tilde{u} \mathbf{a}_{1}+\tilde{v} \mathbf{a}_{2}$, where $\mathbf{v}$ is the velocity vector. The velocity vector $\left(u_{\lambda}, u_{\theta}\right)$ in spherical LAT/LON coordinates $(\lambda, \theta)$ and contravariant velocity vector are related by

$$
\left[\begin{array}{l}
u_{\lambda} \\
u_{\theta}
\end{array}\right]=\mathbf{J}\left[\begin{array}{l}
\tilde{u} \\
\tilde{v}
\end{array}\right]
$$

where $\mathbf{J}$ is a $2 \times 2$ matrix

$$
\mathbf{J}=R\left[\begin{array}{cc}
\cos \theta & 0 \\
0 & 1
\end{array}\right]\left[\begin{array}{ll}
\frac{\partial \lambda}{\partial \xi} & \frac{\partial \lambda}{\partial \eta} \\
\frac{\partial \theta}{\partial \xi} & \frac{\partial \theta}{\partial \eta}
\end{array}\right] .
$$

The metric tensor $G_{i j}$ for local curvilinear grid is then obtained by $G_{i j}=\mathbf{J}^{\mathrm{T}} \mathbf{J}$, and $G_{i j}$ has the same expression for all six patches $[24]$ as

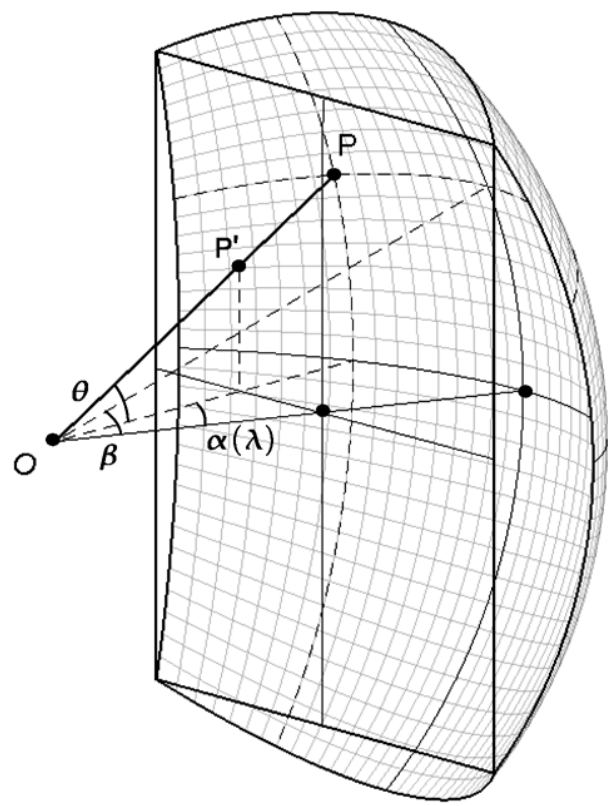

Fig. 2. Projection relation for patch one. 


$$
G_{i j}=\frac{1}{\rho^{4} \cos ^{2} \alpha \cos ^{2} \beta}\left[\begin{array}{cc}
1+\tan ^{2} \alpha & -\tan \alpha \tan \beta \\
-\tan \alpha \tan \beta & 1+\tan ^{2} \beta
\end{array}\right],
$$

where $\rho^{2}=1+\tan ^{2} \alpha+\tan ^{2} \beta$, and $\sqrt{G}=\sqrt{\operatorname{det}\left(G_{i j}\right)}=\rho^{-3} \cos ^{-2} \alpha \cos ^{-2} \beta$ is the Jacobian of transformation.

The covariant and contravariant components of velocity vector are related by

$$
\left[\begin{array}{c}
\tilde{u} \\
\tilde{v}
\end{array}\right]=G^{i j}\left[\begin{array}{l}
u \\
v
\end{array}\right],
$$

where $G^{i j}$ is the inverse of the metric tensor, $G^{i j}=\left(G_{i j}\right)^{-1}$.

\subsection{Shallow water equations on cubed-sphere}

The shallow water equations with vector invariant form of the momentum equation are adopted in present model. In the local non-orthogonal curvilinear grid on each patch, the shallow water equations can be expressed in flux form as $[25,29,23]$

$$
\left\{\begin{array}{l}
\frac{\partial h}{\partial t}=-\frac{1}{\sqrt{G}}\left[\frac{\partial(\sqrt{G} \tilde{u} h)}{\partial \xi}+\frac{\partial(\sqrt{G} \tilde{\partial} h)}{\partial \eta}\right], \\
\frac{\partial u}{\partial t}=\sqrt{G} \tilde{v}(\zeta+f)-\frac{\partial}{\partial \xi}[\Phi+K], \\
\frac{\partial v}{\partial t}=-\sqrt{G} \tilde{u}(\zeta+f)-\frac{\partial}{\partial \eta}[\Phi+K],
\end{array}\right.
$$

where $\Phi=g\left(h+h_{\mathrm{s}}\right)$ is geopotential, $g$ is gravitational constant, $h$ is the height of the fluid over the bottom mountain, $h_{\mathrm{s}}$ is the height of the bottom mountain, $K=\frac{1}{2} \mathbf{v} \cdot \mathbf{v}=\frac{1}{2}(u \tilde{u}+v \tilde{v})$ is the kinetic energy, $f$ the Coriolis parameter defined as $f=2 \Omega \sin \theta, \Omega=7.292 \times 10^{-5} \mathrm{~s}^{-1}$ is the rotation speed of the Earth and $\zeta$ is relative vorticity defined as $\frac{1}{\sqrt{G}}\left(\frac{\partial v}{\partial \xi}-\frac{\partial u}{\partial \eta}\right)$.

Eq. (6) can be re-written as [23],

$$
\frac{\partial \mathbf{Q}}{\partial t}+\frac{\partial \mathbf{E}}{\partial \xi}+\frac{\partial \mathbf{F}}{\partial \eta}=\mathbf{S}
$$

where $\quad \mathbf{Q}=\left[\begin{array}{lll}\sqrt{G} h, & u, & v\end{array}\right]^{\mathrm{T}}, \quad \mathbf{E}=\left[\begin{array}{lll}\sqrt{G} h \tilde{u}, & \Phi+K, & 0\end{array}\right]^{\mathrm{T}}, \quad \mathbf{F}=\left[\begin{array}{lll}\sqrt{G} h \tilde{v}, & 0, & \Phi+K\end{array}\right]^{\mathrm{T}}, \quad$ and $\quad \mathbf{S}=$ $[0, \sqrt{G} \tilde{v}(f+\zeta),-\sqrt{G} \tilde{u}(f+\zeta)]^{\mathrm{T}}$. From (5) and considering the relation $G^{12}=G^{21}=\bar{G}$, the flux terms can be expressed as

$$
\mathbf{E}=\left[\begin{array}{c}
\sqrt{G} h\left(G^{11} u+\bar{G} v\right) \\
g\left(h+h_{\mathrm{s}}\right)+\frac{1}{2} G^{11} u^{2}+\bar{G} u v+\frac{1}{2} G^{22} v^{2} \\
0
\end{array}\right]
$$

and

$$
\mathbf{F}=\left[\begin{array}{c}
\sqrt{G} h\left(\bar{G} u+G^{22} v\right) \\
0 \\
g\left(h+h_{\mathrm{s}}\right)+\frac{1}{2} G^{11} u^{2}+\bar{G} u v+\frac{1}{2} G^{22} v^{2}
\end{array}\right] .
$$

\section{CIP/Multi-moment FVM in two dimensions}

In our multi-moment numerical model, more than one kind of moments are defined and updated independently in time. A high-order scheme can be constructed on a more localized stencil than the traditional singlemoment methods. For the numerical model on the cubed-sphere grid, our scheme is more convenient to maintain the numerical conservation of the mass and deal with the boundaries between the different patches. In the present study, we choose two kinds of moments, i.e. point value (PV) and volume-integrated average (VIA), as the model variables. 
For control volume $\mathcal{C}_{i j}$ shown in Fig. 3 , the moments for a field variable $\psi(\xi, \eta)$ are defined as

- The point value (PV moment)

$$
\begin{aligned}
& \bar{P}_{i \pm \frac{1}{2 j \pm \frac{1}{2}}}=\psi\left(\xi_{i \pm \frac{1}{2}}, \eta_{j \pm \frac{1}{2}}\right), \quad \text { at the four vertices, } \\
& \bar{P}_{i j \pm \frac{1}{2}}=\psi\left(\xi_{i}, \eta_{j \pm \frac{1}{2}}\right), \quad \text { at the midpoints of two } \xi \text { edges, } \\
& \bar{P}^{{ }^{P} \psi_{i \pm \frac{1}{2} j}}=\psi\left(\xi_{i \pm \frac{1}{2}}, \eta_{j}\right), \quad \text { at the midpoints of two } \eta \text { edges }
\end{aligned}
$$

- The volume-integrated average (VIA moment)

$$
\bar{V}_{i j}=\frac{1}{\left|V_{i j}\right|} \int_{\mathcal{C}_{i j}} \psi(\xi, \eta) \mathrm{d} v
$$

where $\left|V_{i j}\right|=\Delta \xi \times \Delta \eta$ is the area of $\mathcal{C}_{i j}$ in terms of the local curvilinear grid, the true area of the control volume is represented by $l_{i j}\left|V_{i j}\right|$ where $l_{i j}=\int_{\mathcal{C}_{i j}} \sqrt{G} \mathrm{~d} v$.

The point value at the center of each element, ${ }^{P} \psi_{i j}$, is not treated as an independent moment, nor a model variable. Instead of being updated independently in time, it is calculated by the above two kinds of moments at every time step. A 2D Simpson's integration rule [1] is used here to compute ${ }^{P_{P}}{ }_{i j}$ as

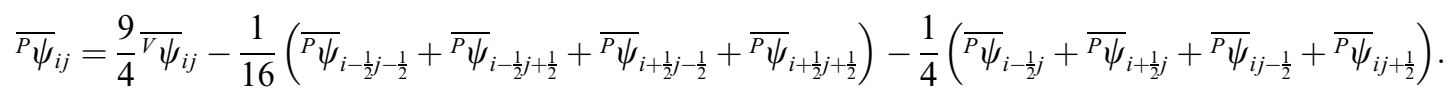

\subsection{Updating the PV moment}

The semi-Lagrangian scheme is often adopted in numerical models to advance the PV moment. It directly applies the characteristic information in the hyperbolic systems, some practical applications are given in $[10,11,37,38,40,41,43]$. In the present model, however, an Eulerian framework is adopted to update the PV moment by solving the local Riemann problem point-wisely. It is more convenient to apply the high-order time integration algorithm and to deal with the source terms in the governing equations cast in a non-orthogonal coordinate system on the cubed-sphere.

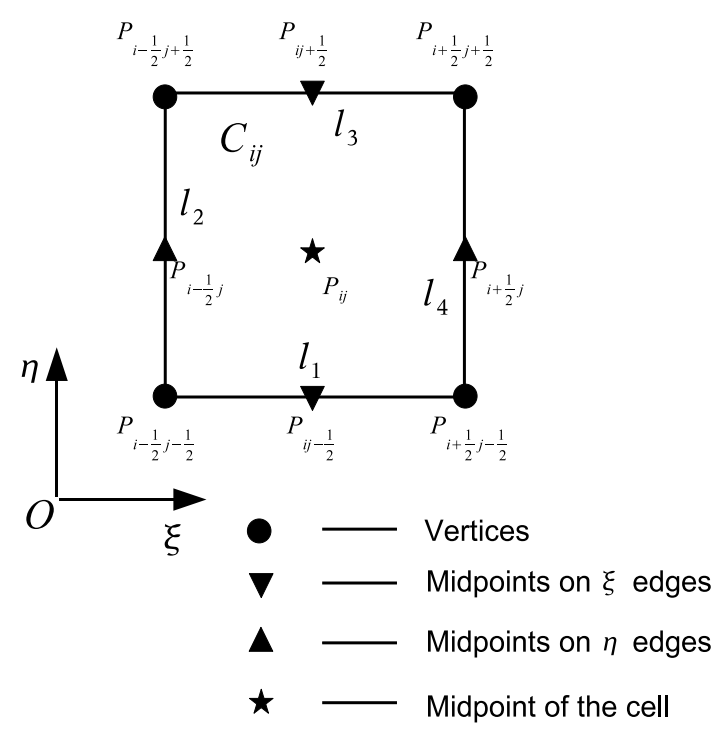

Fig. 3. The control volume $\left(\mathcal{C}_{i j}\right)$ for multi-moment discretization. 
With the terms related to the topographic effect separated from the flux, the governing equations (7) are recast into

$$
\frac{\partial \mathbf{Q}}{\partial t}+\frac{\partial \mathbf{E}^{\prime}}{\partial \xi}+\frac{\partial \mathbf{F}^{\prime}}{\partial \eta}+\mathbf{S}_{T}=\mathbf{S}
$$

where $\mathbf{E}^{\prime}$ and $\mathbf{F}^{\prime}$ denote the flux functions without the topographic terms which are incorporated into the additional source terms $\mathbf{S}_{T}=\left[0, \frac{\partial}{\partial \xi}\left(g h_{\mathrm{s}}\right), \frac{\partial}{\partial \eta}\left(g h_{\mathrm{s}}\right)\right]^{\mathrm{T}}$.

The PV moments are updated by computing (13) point-wisely at $\left(\xi_{i-\frac{1}{2}}, \eta_{j-\frac{1}{2}}\right),\left(\xi_{i}, \eta_{j-\frac{1}{2}}\right)$ and $\left(\xi_{i-\frac{1}{2}}, \eta_{j}\right)$. The key point here is how to evaluate the spatial derivatives of the flux functions.

We re-write the derivatives of the flux functions into

$$
\frac{\partial \mathbf{E}^{\prime}}{\partial \xi}=\mathbf{A} \frac{\partial \mathbf{Q}}{\partial \xi}+\mathbf{S}_{C \xi}
$$

and

$$
\frac{\partial \mathbf{F}^{\prime}}{\partial \eta}=\mathbf{B} \frac{\partial \mathbf{Q}}{\partial \eta}+\mathbf{S}_{C \eta}
$$

where $\mathbf{A}$ and $\mathbf{B}$ are the Jacobian matrices of fluxes $\mathbf{E}^{\prime}$ and $\mathbf{F}^{\prime}$,

$$
\mathbf{A}=\frac{\partial \mathbf{E}^{\prime}}{\partial \mathbf{Q}}=\left[\begin{array}{ccc}
G^{11} u+\bar{G} v & \sqrt{G} G^{11} h & \sqrt{G} \bar{G} h \\
\frac{g}{\sqrt{G}} & G^{11} u+\bar{G} v & \bar{G} u+G^{22} v \\
0 & 0 & 0
\end{array}\right]
$$

and

$$
\mathbf{B}=\frac{\partial \mathbf{F}^{\prime}}{\partial \mathbf{Q}}=\left[\begin{array}{ccc}
\bar{G} u+G^{22} v & \sqrt{G} \bar{G} h & \sqrt{G} G^{22} h \\
0 & 0 & 0 \\
\frac{g}{\sqrt{G}} & G^{11} u+\bar{G} v & \bar{G} u+G^{22} v
\end{array}\right] .
$$

Since $\sqrt{G}$ and the matrix $G^{i j}$ are the functions of the space location $(\xi, \eta)$, we have two extra terms $\mathbf{S}_{C \xi}$ and $\mathbf{S}_{C \eta}$ written as

$$
\mathbf{S}_{C \xi}=\left[\begin{array}{c}
\sqrt{G} h u \frac{\partial G^{11}}{\partial \xi}+\sqrt{G} h v \frac{\partial \bar{G}}{\partial \xi} \\
-\frac{g h}{\sqrt{G}} \frac{\partial \sqrt{G}}{\partial \xi}+\frac{1}{2} u^{2} \frac{G^{11}}{\partial \xi}+u v \frac{\partial \bar{G}}{\partial \xi}+\frac{1}{2} v^{2} \frac{\partial G^{22}}{\partial \xi} \\
0
\end{array}\right]
$$

and

$$
\mathbf{S}_{C \eta}=\left[\begin{array}{c}
\sqrt{G} h u \frac{\partial \bar{G}}{\partial \eta}+\sqrt{G} h v \frac{\partial G^{22}}{\partial \eta} \\
0 \\
-\frac{g h}{\sqrt{G}} \frac{\partial \sqrt{G}}{\partial \eta}+\frac{1}{2} u^{2} \frac{G^{11}}{\partial \eta}+u v \frac{\partial \bar{G}}{\partial \eta}+\frac{1}{2} v^{2} \frac{\partial G^{22}}{\partial \eta}
\end{array}\right] .
$$

Because the first-order derivatives of the piecewise interpolation functions are not continuous at cell boundaries where the PV moments need to be predicted, we use a local Lax-Friedrichs splitting scheme in terms of the derivatives, which is similar to the scheme used in $[24,23]$ and the detailed expressions can be referred to Appendix in [23]. The flux derivative in $\xi$-direction is then calculated by

$$
\frac{\partial \mathbf{E}^{\prime}}{\partial \xi}=\frac{1}{2}\left[\mathbf{A}\left(\frac{\partial \mathbf{Q}^{+}}{\partial \xi}+\frac{\partial \mathbf{Q}^{-}}{\partial \xi}\right)+a\left(\frac{\partial \mathbf{Q}^{+}}{\partial \xi}-\frac{\partial \mathbf{Q}^{-}}{\partial \xi}\right)\right]+\mathbf{S}_{C \xi},
$$

where superscriptions "+" and "-" denote the values calculated using the interpolations respectively constructed on the left and the right stencils.

The parameter $a$ is chosen as the maximum of the absolute values of the characteristic velocities at the corresponding point. From $\left|\mathbf{A}-\lambda_{\xi} \mathbf{I}\right|=0$, we obtain the characteristic velocities in $\xi$-direction as $\lambda_{\xi}=\tilde{u} \pm \sqrt{G^{11} g h}$ (see [23], Appendix), thus $a=|\tilde{u}|+\sqrt{G^{11} g h}$. 
Similarly, in $\eta$-direction, the flux derivative is

$$
\frac{\partial \mathbf{F}^{\prime}}{\partial \eta}=\frac{1}{2}\left[\mathbf{B}\left(\frac{\partial \mathbf{Q}^{+}}{\partial \eta}+\frac{\partial \mathbf{Q}^{-}}{\partial \eta}\right)+b\left(\frac{\partial \mathbf{Q}^{+}}{\partial \eta}-\frac{\partial \mathbf{Q}^{-}}{\partial \eta}\right)\right]+\mathbf{S}_{C \eta}
$$

and the parameter $b$ equals to $|\tilde{v}|+\sqrt{G^{22} g h}$ which is obtained from the eigenvalues of Jacobian matrix B.

To calculate the derivatives in (20) and (21), a four-point asymmetric difference formulation is adopted. For the PVs defined at the vertices of the control volume, i.e. at point $\left(\xi_{i-\frac{1}{2}}, \eta_{j-\frac{1}{2}}\right)$ in Fig. 4 , the derivatives of any field variable $\psi$ can be computed by

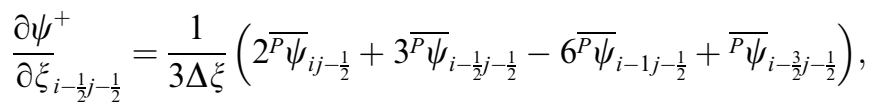

$$
\begin{aligned}
& \frac{\partial \psi^{-}}{\partial \xi_{i-\frac{1}{2} j-\frac{1}{2}}}=\frac{1}{3 \Delta \xi}\left(-\bar{P}_{i+\frac{1}{2} j-\frac{1}{2}}+6^{P} \bar{\psi}_{i j-\frac{1}{2}}-3^{P} \bar{\psi}_{i-\frac{1}{2} j-\frac{1}{2}}-2^{P} \bar{\psi}_{i-1 j-\frac{1}{2}}\right) \text {, } \\
& \frac{\partial \psi^{+}}{\partial \eta_{i-\frac{1}{2} j-\frac{1}{2}}}=\frac{1}{3 \Delta \eta}\left(2^{\bar{P} \psi_{i-\frac{1}{2} j}}+3^{\bar{P}}{ }_{i-\frac{1}{2} j-\frac{1}{2}}-6^{P} \bar{\psi}_{i-\frac{1}{2} j-1}+\bar{P}_{i-\frac{1}{2} j-\frac{3}{2}}\right), \\
& \frac{\partial \psi^{-}}{\partial \eta_{i-\frac{1}{2} j-\frac{1}{2}}}=\frac{1}{3 \Delta \eta}\left(-\bar{P}_{i-\frac{1}{2} j+\frac{1}{2}}+6 \bar{P}^{P} \psi_{i-\frac{1}{2} j}-3^{P} \bar{\psi}_{i-\frac{1}{2} j-\frac{1}{2}}-2^{P} \bar{\psi}_{i-\frac{1}{2} j-1}\right) .
\end{aligned}
$$

For the midpoints of the boundary edges, when one evaluates $\frac{\partial \psi}{\partial \eta}$ at $\left(\xi_{i}, \eta_{j-\frac{1}{2}}\right)$ and $\frac{\partial \psi}{\partial \xi}$ at $\left(\xi_{i-\frac{1}{2}}, \eta_{j}\right)$ (see Fig. 4), a similar four-point formulation requires the point value, ${ }^{{ }^{P} \psi_{i j}}$, at the center of the control volume. We compute $\bar{P}_{i j}$ from the known moments by (12).

On the other hand, the flux derivatives aligning the grid line, i.e. $\frac{\partial \mathbf{E}^{\prime}}{\partial \xi}$ at $\left(\xi_{i}, \eta_{j-\frac{1}{2}}\right)$ and $\frac{\partial \mathbf{F}^{\prime}}{\partial \eta}$ at $\left(\xi_{i-\frac{1}{2}}, \eta_{j}\right)$, are calculated by applying the Simpson's rule [1] with the flux derivatives at the vertices given above,

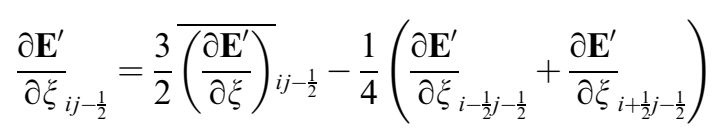

and

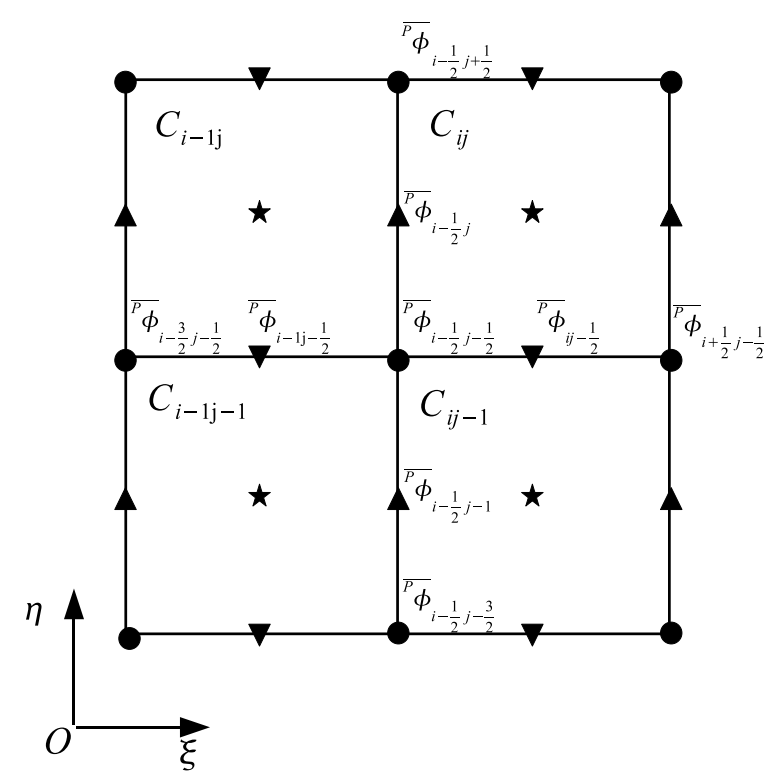

Fig. 4. Reconstruction stencils for evaluating the derivatives of the state variables. 


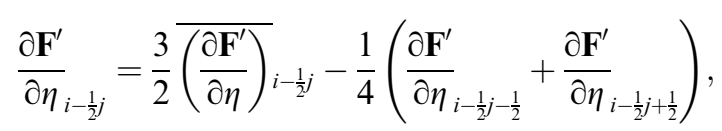

where

$$
\overline{\left(\frac{\partial \mathbf{E}^{\prime}}{\partial \xi}\right)_{i j-\frac{1}{2}}}=\frac{\mathbf{E}_{i+\frac{1}{2} j-\frac{1}{2}}^{\prime}-\mathbf{E}_{i-\frac{1}{2} j-\frac{1}{2}}^{\prime}}{\Delta \xi}
$$

and

$$
{\overline{\left(\frac{\partial \mathbf{F}^{\prime}}{\partial \eta}\right)_{i-\frac{1}{2} j}}}=\frac{\mathbf{F}_{i-\frac{1}{2} j+\frac{1}{2}}^{\prime}-\mathbf{F}_{i-\frac{1}{2} j-\frac{1}{2}}^{\prime}}{\Delta \eta}
$$

are the line-integrated averages along the boundary edges of the control volume $\mathcal{C}_{i j}$ for the corresponding flux derivatives respectively.

Special attention must be paid around the patch boundaries in the cubed-sphere grid. We include one-layer ghost cells along the patch boundaries. Fig. 5 shows the case for the boundary edge between patch one and patch four. The PVs on the points denoted by the solid markers should be calculated by the known PVs and VIAs from the neighboring patches. Firstly the positions of the ghost points are determined by the geometrical relation. Since the position is fixed, it is calculated at the initial step and stored as $(\xi, \eta, i, j, k)$ which denotes the ghost point belonging to the control volume $\mathcal{C}_{i j}$ of the $k$ th patch and located at $(\xi, \eta)$ in local curvilinear coordinates. Then the ghost PVs are calculated from the interpolation

$$
\begin{aligned}
\Psi_{i j}(\xi, \eta)= & c_{i j}^{00}+c_{i j}^{10}\left(\xi-\xi_{i-\frac{1}{2}}\right)+c_{i j}^{01}\left(\eta-\eta_{j-\frac{1}{2}}\right)+c_{i j}^{11}\left(\xi-\xi_{i-\frac{1}{2}}\right)\left(\eta-\eta_{j-\frac{1}{2}}\right)+c_{i j}^{20}\left(\xi-\xi_{i-\frac{1}{2}}\right)^{2} \\
& +c_{i j}^{02}\left(\eta-\eta_{j-\frac{1}{2}}\right)^{2}+c_{i j}^{21}\left(\xi-\xi_{i-\frac{1}{2}}\right)^{2}\left(\eta-\eta_{j-\frac{1}{2}}\right)+c_{i j}^{12}\left(\xi-\xi_{i-\frac{1}{2}}\right)\left(\eta-\eta_{j-\frac{1}{2}}\right)^{2} \\
& +c_{i j}^{22}\left(\xi-\xi_{i-\frac{1}{2}}\right)^{2}\left(\eta-\eta_{j-\frac{1}{2}}\right)^{2} .
\end{aligned}
$$

The coefficients of (30) are determined by the nine independent moments (including eight PVs and one VIA) of the corresponding cell on the neighboring patch. The detailed expressions of the coefficients are given in the appendix. Provided the values at these ghost points, we can compute the whole patch (including the boundary edges) by the numerical procedure described above. As we will see from the numerical tests later, using interpolation (30) to communicate data across patch boundaries does not degrade the numerical accuracy of the whole model.

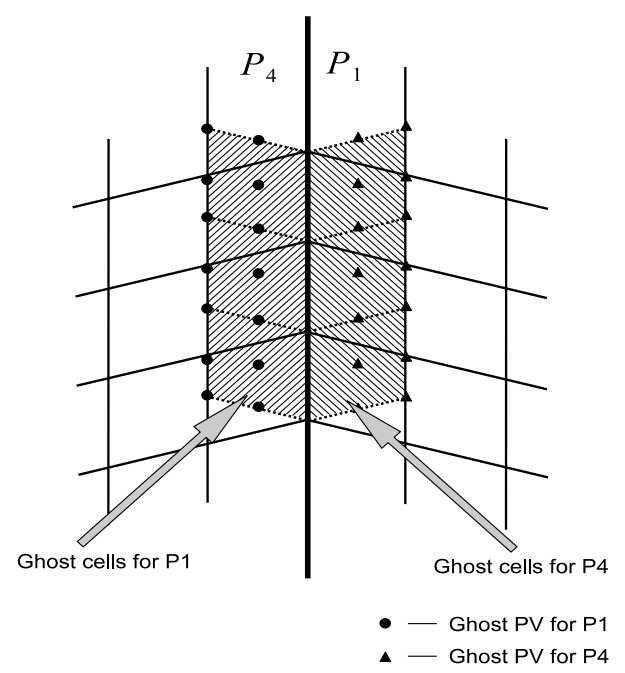

Fig. 5. The ghost cells for the boundary edge between patch one and patch four. 
During the computation, the PVs on the boundaries will be updated in different patches, for example, the point denoted by solid circle in Fig. 6 is located on the boundary edge between patch one and patch four. The PV moment defined at this point can be updated on patch one or four with the ghost cells built from either side. In general, the results obtained from the different patches are not identical. In the present model, a simple algebraic averaging of the edge-point values computed from different patches is adopted. For scalar $h$, the PV is computed by averaging the different results directly. For the velocity vector, the projection relations between different local coordinates should also be taken into account in this averaging procedure. Similarly, to update the PV moment defined on the vertices of the inscribed cube, this averaging will involve three adjacent patches.

For the problems involving a bottom topography, the "exact C-property", which means the stationary state should be exactly preserved when the numerical model is applied to the stationary case, i.e. $h+h_{\mathrm{s}}=$ constant and $h \mathbf{v}=0$, is of particular importance [3,42]. We have made two manipulations in the numerical model to assure the numerical balance at the discretization level. The first one is to modify the difference terms of continuity equation. Considering that fluid height $h$, the derivatives of the mountain height and $\sqrt{G}$ remain continuous at the control volume boundary, the following relations hold

$$
\left\{\begin{array}{l}
\frac{\partial(\sqrt{G} h)^{+}}{\partial \xi}-\frac{\partial(\sqrt{G} h)^{-}}{\partial \xi}=\sqrt{G}\left(\frac{\partial h_{t}^{+}}{\partial \xi}-\frac{\partial h_{t}^{-}}{\partial \xi}\right), \\
\frac{\partial(\sqrt{G} h)^{+}}{\partial \eta}-\frac{\partial(\sqrt{G} h)^{-}}{\partial \eta}=\sqrt{G}\left(\frac{\partial h_{t}^{+}}{\partial \eta}-\frac{\partial h^{-}}{\partial \eta}\right),
\end{array}\right.
$$

where $h_{\mathrm{t}}=h+h_{\mathrm{s}}$ is the total height.

In the numerical model, we use the right-hand side formulations in the above expressions when discretising the continuity equation (the first equations in (20) and (21)), which assure the fluid height calculated from continuity equation remains unchanging when $h_{\mathrm{t}}=$ constant. The second modification is to the topographic source term in the momentum equations (the second and third equations in (20) and (21)). The derivative of the mountain height $\frac{\partial\left(g h_{s}\right)}{\partial \xi}$ is calculated as

$$
\frac{\partial\left(g h_{\mathrm{s}}\right)}{\partial \xi}=\frac{1}{2} \frac{g}{\sqrt{G}}\left[\frac{\partial\left(\sqrt{G} h_{\mathrm{s}}\right)^{+}}{\partial \xi}+\frac{\partial\left(\sqrt{G} h_{\mathrm{s}}\right)^{-}}{\partial \xi}\right]-\frac{g h_{\mathrm{s}}}{\sqrt{G}} \frac{\partial \sqrt{G}}{\partial \xi},
$$

at points $\left(\xi_{i-\frac{1}{2}}, \eta_{j-\frac{1}{2}}\right),\left(\xi_{i-\frac{1}{2}}, \eta_{j}\right)$ (see Fig. 4), and the "+" and "- " derivatives are calculated by (22) and (23). At point $\left(\xi_{i}, \eta_{j-\frac{1}{2}}\right)$ the derivative is computed by

$$
\frac{\partial\left(g h_{\mathrm{s}}\right)}{\partial \xi}=\frac{3}{2}{\frac{\overline{\partial\left(g h_{\mathrm{s}}\right)}}{\partial \xi}}_{i j-\frac{1}{2}}-\frac{1}{4}\left({\frac{\partial\left(g h_{\mathrm{s}}\right)}{\partial \xi_{i-\frac{1}{2} j-\frac{1}{2}}}}+{\frac{\partial\left(g h_{\mathrm{s}}\right)}{\partial \xi_{i+\frac{1}{2} j-\frac{1}{2}}}}\right),
$$

where

$$
\frac{\overline{\partial\left(g h_{\mathrm{s}}\right)}}{\partial \xi}{ }_{i j-\frac{1}{2}}=\frac{\left(g h_{\mathrm{s}}\right)_{i+\frac{1}{2} j-\frac{1}{2}}-\left(g h_{\mathrm{s}}\right)_{i-\frac{1}{2} j-\frac{1}{2}}}{\Delta \xi} .
$$

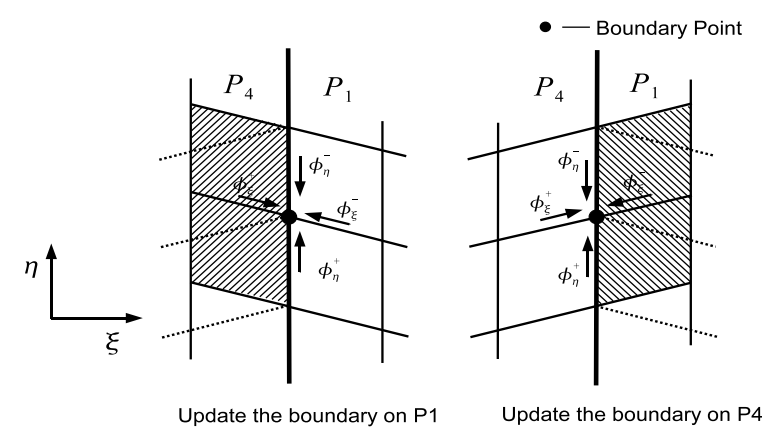

Fig. 6. The derivative Riemann problem on the boundary edge. 
Similarly, the source term $\frac{\partial\left(g h_{\mathrm{s}}\right)}{\partial \eta}$ is calculated as

$$
\frac{\partial\left(g h_{\mathrm{s}}\right)}{\partial \eta}=\frac{1}{2} \frac{g}{\sqrt{G}}\left[\frac{\partial\left(\sqrt{G} h_{\mathrm{s}}\right)^{+}}{\partial \eta}+\frac{\partial\left(\sqrt{G} h_{\mathrm{s}}\right)^{-}}{\partial \eta}\right]-\frac{g h_{\mathrm{s}}}{\sqrt{G}} \frac{\partial \sqrt{G}}{\partial \eta},
$$

at points $\left(\xi_{i-\frac{1}{2}}, \eta_{j-\frac{1}{2}}\right)$ and $\left(\xi_{i}, \eta_{j-\frac{1}{2}}\right)$, and the "+" and "-" derivatives are calculated by (24) and (25). At point $\left(\xi_{i-\frac{1}{2}}, \eta_{j}\right)$ the derivative is computed by

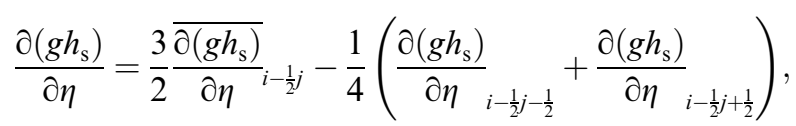

where

$$
\frac{\overline{\partial\left(g h_{\mathrm{s}}\right)}}{\partial \eta}{ }_{i-\frac{1}{2} j}=\frac{\left(g h_{\mathrm{s}}\right)_{i-\frac{1}{2} j+\frac{1}{2}}-\left(g h_{\mathrm{s}}\right)_{i-\frac{1}{2} j-\frac{1}{2}}}{\Delta \eta} .
$$

In the second modification, we use the same formulation to calculate the derivatives of the fluid height and the mountain height, then the derivatives of the fluid height $h$ in flux terms can be rewritten into derivatives of the total hight $h_{\mathrm{t}}$ after being combined with derivatives of mountain height $h_{\mathrm{s}}$ in topographic source terms. It is easy to show that with these modifications the "exact C-property" of the topographic source terms is always satisfied.

The derivatives in source term $\mathbf{S}(\mathbf{Q})$ are calculated by a five-point central difference formulation.

\subsection{Updating the VIA moment}

The VIA moment is updated by a flux-form finite volume formulation at each time step to guarantee the numerical conservation. For control volume $\mathcal{C}_{i j}$ shown in Fig. 3, we denote the boundary edges by $l_{1} \equiv \overline{P_{i-\frac{1}{2} j-\frac{1}{2}} P_{i+\frac{1}{2} j-\frac{1}{2}}}, l_{2} \equiv \overline{P_{i-\frac{1}{2} j-\frac{1}{2}} P_{i-\frac{1}{2} j+\frac{1}{2}}}, l_{3} \equiv \overline{P_{i-\frac{1}{2} j+\frac{1}{2}} P_{i+\frac{1}{2} j+\frac{1}{2}}}$ and $l_{4} \equiv \overline{P_{i+\frac{1}{2} j-\frac{1}{2}} P_{i+\frac{1}{2} j+\frac{1}{2}}}$.

To update the VIA, (7) is integrated over the control volume. With the application of the Gauss theorem, a semi-discretised formulation is written as

$$
\frac{\partial}{\partial t}\left(\overline{V q}_{m}\right)_{i j}=-\frac{1}{\left|V_{i j}\right|}\left(\sum_{k=1}^{4} \int_{l_{k}} \mathbf{T}_{m} \cdot \mathbf{n}_{l_{k}} \mathrm{~d} l+\int_{\mathcal{C}_{i j}} s_{m} \mathrm{~d} v\right) \quad(m=1,2,3),
$$

where the equations of $m=1,2,3$ denote the continuity equation and the momentum equations in $\xi$ and $\eta$ directions, $q_{m}, e_{m}, f_{m}$ and $s_{m}$ are used to represent the components of vectors $\mathbf{Q}, \mathbf{E}, \mathbf{F}, \mathbf{S}$ in (7) respectively. $\mathbf{T}_{m}=\left(e_{m}, f_{m}\right)$ is the corresponding flux vector with $e_{m}$ and $f_{m}$ being the components in $\xi$ and $\eta$ directions. $\mathbf{n}_{l_{k}}$ is the outward unit normal vector of edge $l_{k}$.

Given the PVs computed through the procedure discussed above, the update of the VIA moment is straightforward. The fluxes crossing boundaries are calculated directly from the PV moment defined on the boundary edges of the control volume. The involved line-integration and surface-integration are computed by the 1D and 2D Simpson's formulas [1].

The line-integrations of numerical flux are calculated as follows:

$$
\begin{aligned}
& \int_{l_{1}} \mathbf{T}_{m} \cdot \mathbf{n}_{l_{1}} \mathrm{~d} l=-\frac{\Delta \xi}{6}\left(f_{m_{i-\frac{1}{2} j-\frac{1}{2}}}+4 f_{m_{i j-\frac{1}{2}}}+f_{m_{i+\frac{1}{2} j-\frac{1}{2}}}\right), \\
& \int_{l_{2}} \mathbf{T}_{m} \cdot \mathbf{n}_{l_{2}} \mathrm{~d} l=-\frac{\Delta \eta}{6}\left(e_{m_{i-\frac{1}{2} j-\frac{1}{2}}}+4 e_{m_{i-\frac{1}{2} j}}+e_{m_{i-\frac{1}{2} j+\frac{1}{2}}}\right), \\
& \int_{l_{3}} \mathbf{T}_{m} \cdot \mathbf{n}_{l_{3}} \mathrm{~d} l=\frac{\Delta \xi}{6}\left(f_{m_{i-\frac{1}{2} j+\frac{1}{2}}}+4 f_{m_{i j+\frac{1}{2}}}+f_{m_{i+\frac{1}{2} j+\frac{1}{2}}}\right), \\
& \int_{l_{4}} \mathbf{T}_{m} \cdot \mathbf{n}_{l_{4}} \mathrm{~d} l=\frac{\Delta \eta}{6}\left(e_{m_{i+\frac{1}{2} j-\frac{1}{2}}}+4 e_{m_{i+\frac{1}{2} j}}+e_{m_{i+\frac{1}{2} j+\frac{1}{2}}}\right)
\end{aligned}
$$


and the surface-integration of the source term is calculated by

$$
\int_{\mathcal{C}_{i j}} s_{m} \mathrm{~d} v=\frac{\Delta \xi \Delta \eta}{36}\left(s_{m_{i-\frac{1}{2} j-\frac{1}{2}}}+s_{m_{i-\frac{1}{2} j+\frac{1}{2}}}+s_{m_{i+\frac{1}{2} j-\frac{1}{2}}}+s_{m_{i+\frac{1}{2} j+\frac{1}{2}}}+4 s_{m_{i-\frac{1}{2} j}}+4 s_{m_{i+\frac{1}{2} j}}+4 s_{m_{i j-\frac{1}{2}}}+4 s_{m_{i j+\frac{1}{2}}}+16 s_{m_{i j}}\right),
$$

where the values of source term at boundary edges of the control volume have been calculated when updating the PV moment, the value at the center of the control volume is computed by calculating the derivatives in source term with the same five-point central difference formulation.

\subsection{Time marching scheme}

We have so far described the spatial discretizations of the evolution equations (13) for the PV and (38) for the VIA moments. With all spatial discretizations accomplished, the evolution equations for both PV and VIA moments can be cast into a form as

$$
\frac{\partial \psi}{\partial t}=\mathcal{L}(\psi)
$$

where $\psi$ denotes any moment, and $\mathcal{L}(\psi)$ represents the spatial discretization formulation.

Provided the value of $\psi$ at time step $t^{n}, \psi^{n}$, the value for the next time step $\psi^{n+1}$ can be computed by a standard 4th-order Runge-Kutta scheme [1] as

$$
\psi^{n+1}=\psi^{n}+\frac{\Delta t}{6}\left(k_{1}+2 k_{2}+2 k_{3}+k_{4}\right)
$$

where

$$
\begin{aligned}
& k_{1}=\mathcal{L}\left(\psi^{n}\right), \\
& k_{2}=\mathcal{L}\left(\psi^{n}+\frac{1}{2} \Delta t k_{1}\right), \\
& k_{3}=\mathcal{L}\left(\psi^{n}+\frac{1}{2} \Delta t k_{2}\right), \\
& k_{4}=\mathcal{L}\left(\psi^{n}+\Delta t k_{3}\right) .
\end{aligned}
$$

In this section, we have described the basic numerical formulations of using the multi-moment to construct shallow water model on the cubed-sphere. Before ending this section, we highlight the major features of the present model as follows.

Two kinds of moments, i.e. PV moment and VIA moment, are treated as the predicted variables in present model. The moments, which are coupled through the local reconstruction based on both kinds of moments, are updated independently by governing equations of different forms, i.e. point-wise differential form (13) for the PV moment and the cell-integrated flux form (38) for VIA moment. The derivatives of the flux functions in (13) are approximated by local derivative Riemann solvers. For the PV moment on the vertices of the control volume, two discontinuous derivatives of independent variables, i.e. $\frac{\partial \psi^{+}}{\partial \xi}$ and $\frac{\partial \psi^{-}}{\partial \xi}$ or $\frac{\partial \psi^{+}}{\partial \eta}$ and $\frac{\partial \psi^{-}}{\partial \eta}$, are calculated from high order multi-moment reconstructions in each direction. Special attention should be paid to the PV moments located at the midpoints of the control volume edges where reconstructions required for the local approximate Riemann solvers in different directions have to been built in different ways. Once the PV moments on the cell boundary are calculated, the fluxes required to update the VIA moment in the finite volume formulation are obtained by numerical quadrature. A multi-step Runge-Kutta scheme is used for time integration to assure the numerical accuracy in time.

Compared to the conventional finite volume method where only one kind of moment is used as the model variable, a multi-moment formulation requires more memory storage space and computational overhead due to the increase in the number of the degrees of freedom (DOF). Similar to other advanced schemes that make reconstructions in terms of locally increased DOF and use multi-step Runge-Kutta integration for high accuracy in time, such as the discontinuous Galerkin method, the current method is more computationally expensive than the conventional methods that make use of simpler reconstructions and single-step updating. 
Nevertheless, it should be notified that the increase in DOF in the present method does not necessarily lead to a more restrictive CFL condition for computational stability that usually applies to a conventional singlemoment scheme when a finer grid is used for higher resolution. Furthermore, the spatial reconstruction is required only for predicting the PV moment, which can be then directly used in the numerical fluxes for updating the VIA moment.

The extension of the present method to 3D is straightforward. A 3D reconstruction can be built with one VIA and 26 PVs within a single-cell. The derivatives of flux function needed to update the PVs at the vertices of a cubic element can be computed through the derivative Riemann problem in each direction respectively, while for the middle points of line segments or surface segments special formulations in terms of the constraints on cell-integrated quantities should be used. With all PVs on the surface of the control volume found, the VIA moment can be advanced by the finite volume formulation.

\section{Numerical tests}

In this section, numerical experiments are carried out to verify the present numerical model. Firstly, advection tests with smooth initial distributions on the cubed sphere are conducted to numerically verify the convergence rate of the present scheme with gradually refined grids. We then present several widely used benchmark tests to evaluate the performance of the numerical model in spherical geometry for both pure advection and shallow water flows. Most of the tests are introduced in Williamson's standard test set [36].

To quantify the numerical errors, the following normalized error measures [36] are used in this section

$$
l_{1} \equiv \frac{\int_{S}\left|\psi-\psi_{t}\right| \mathrm{d} s}{\int_{S}\left|\psi_{t}\right| \mathrm{d} s}, \quad l_{2} \equiv \frac{\left[\int_{S}\left(\psi-\psi_{t}\right)^{2} \mathrm{~d} s\right]^{\frac{1}{2}}}{\left(\int_{S} \psi_{t}^{2} \mathrm{~d} s\right)^{\frac{1}{2}}}, \quad l_{\infty} \equiv \frac{\max \left|\psi-\psi_{t}\right|}{\max \left|\psi_{t}\right|},
$$

where $S$ is the computational domain, $\psi$ is the numerical result and $\psi_{t}$ is the exact solution.

\subsection{Advection equation tests}

\subsubsection{Convergence rate evaluation}

The convergence rate of present scheme on the cubed sphere is examined by numerical tests with an advection problem on sphere. The governing equation in this case is written as (continuity equation in (7))

$$
\frac{\partial(\sqrt{G} h)}{\partial t}+\frac{\partial(\tilde{u} \sqrt{G} h)}{\partial \xi}+\frac{\partial(\tilde{v} \sqrt{G} h)}{\partial \eta}=0 .
$$

To check the performance of the present scheme in different flow directions, the initial condition is given in rotated LAT/LON coordinates as

$$
h\left(\lambda^{\prime}, \theta^{\prime}\right)=\cos ^{p} \theta^{\prime} \sin k \lambda^{\prime},
$$

where the origin of rotated coordinates is located at $(0, \alpha)$ in the original LAT/LON coordinates. By using the formulation given in [17], the $\left(\lambda^{\prime}, \theta^{\prime}\right)$ is calculated from $(\lambda, \theta)$ as

$$
\left\{\begin{array}{l}
\lambda^{\prime}=\arctan \left(\frac{\cos \theta \sin \lambda}{\cos \theta \cos \lambda \cos \alpha+\sin \theta \sin \alpha}\right), \\
\theta^{\prime}=\arcsin (\sin \theta \cos \alpha-\cos \theta \cos \lambda \sin \alpha) .
\end{array}\right.
$$

A divergence-free velocity field is given by [36]

$$
\left\{\begin{array}{l}
u_{\lambda}=u_{0}(\cos \theta \cos \alpha+\sin \theta \cos \lambda \sin \alpha) \\
u_{\theta}=-u_{0} \sin \lambda \sin \alpha
\end{array}\right.
$$

where $u_{0}=2 \pi R /(12$ days $)$ and the parameter $\alpha$ represents the angle between the rotation axis and polar axis of the Earth.

In this test, we specify $p=4$ and $k=4$. The CFL number of 0.25 is adopted. The tests in different flow directions of $\alpha=0, \alpha=\frac{\pi}{4}$ and $\alpha=\frac{\pi}{2}$ are carried out on grids of different resolutions. Because of the symmetry 
of the cubed-sphere grid, the results of the advections along the equator and the meridian produce identical numerical results. For a cubed-sphere, the case of $\alpha=\frac{\pi}{4}$ is of much interest. In this case, the flow trajectory passes two complete boundaries and four vertices of the inscribed cube. The results of the tests on different grid resolutions and in different flow directions are shown in Table 1-3.

It is revealed that the numerical treatments along the patch boundaries do not degrade the numerical accuracy. We achieved the 4th-order convergence rate in all tests along three different flow directions.

\subsubsection{Cosine bell advection test}

Being the first test of Williamson's standard set [36], the solid rotation of a cosine bell on the sphere was computed. The initial height field is given as

$$
h= \begin{cases}\left(\frac{h_{0}}{2}\right)\left(1+\cos \frac{\pi r}{r_{0}}\right) & \text { if } r<r_{0}, \\ 0 & \text { otherwise }\end{cases}
$$

where $r$ is the great circle distance between point $(\lambda, \theta)$ and the initial center $\left(\lambda_{0}, \theta_{0}\right)=\left(\frac{3 \pi}{2}, 0\right)$. Other constants are specified as $h_{0}=1000 \mathrm{~m}$ and $r_{0}=\frac{R}{3}$.

The same divergence-free flow field is given as (51) in the previous test. It is also suggested [36] that the advections in different rotating directions should be carried out in this test. Again, three directions of $\alpha=0, \alpha=\frac{\pi}{4}$ and $\alpha=\frac{\pi}{2}$ are chosen here. The grid resolution of $32 \times 32 \times 6$ and the CFL number of 0.5 are adopted.

The height field advected by the flow in the direction of $\alpha=\frac{\pi}{4}$ after one revolution is given in Fig. 7. The normalized errors for the advections in different directions are given in Table 4. Compared with the results calculated by the semi-Lagrangian scheme on both the gnomonic cubic grid (see [19], Fig. 7) and conformal

Table 1

Convergence rate of CIP/MM advection scheme on the cubed-sphere in the direction of $\alpha=0$

\begin{tabular}{|c|c|c|c|c|c|c|}
\hline \multirow[t]{2}{*}{ Grid } & \multicolumn{2}{|l|}{$l_{1}$} & \multicolumn{2}{|l|}{$l_{2}$} & \multicolumn{2}{|l|}{$l_{\infty}$} \\
\hline & Error & Order & Error & Order & Error & Order \\
\hline $10 \times 10 \times 6$ & $1.5837 \mathrm{e}-2$ & - & $1.8592 \mathrm{e}-2$ & - & $1.8731 \mathrm{e}-2$ & - \\
\hline $20 \times 20 \times 6$ & $1.2281 \mathrm{e}-3$ & 3.69 & $1.4410 \mathrm{e}-3$ & 3.67 & $1.5120 \mathrm{e}-3$ & 3.63 \\
\hline $40 \times 40 \times 6$ & $8.2611 \mathrm{e}-5$ & 3.89 & $9.7250 \mathrm{e}-5$ & 3.89 & $1.0210 \mathrm{e}-4$ & 3.89 \\
\hline $80 \times 80 \times 6$ & $5.2864 \mathrm{e}-6$ & 3.97 & $6.2334 \mathrm{e}-6$ & 3.96 & $6.5316 \mathrm{e}-6$ & 3.97 \\
\hline
\end{tabular}

Table 2

Same as Table 1 , but in the direction of $\alpha=\frac{\pi}{4}$

\begin{tabular}{|c|c|c|c|c|c|c|}
\hline \multirow[t]{2}{*}{ Grid } & \multicolumn{2}{|l|}{$l_{1}$} & \multicolumn{2}{|l|}{$l_{2}$} & \multicolumn{2}{|l|}{$l_{\infty}$} \\
\hline & Error & Order & Error & Order & Error & Order \\
\hline $10 \times 10 \times 6$ & $1.6827 \mathrm{e}-2$ & - & $1.6924 \mathrm{e}-2$ & - & $1.3349 \mathrm{e}-2$ & - \\
\hline $20 \times 20 \times 6$ & $1.1879 \mathrm{e}-3$ & 3.82 & $1.1993 \mathrm{e}-3$ & 3.82 & $9.3661 \mathrm{e}-4$ & 3.83 \\
\hline $40 \times 40 \times 6$ & $7.7420 \mathrm{e}-5$ & 3.94 & $7.8026 \mathrm{e}-5$ & 3.94 & $6.1634 \mathrm{e}-5$ & 3.93 \\
\hline $80 \times 80 \times 6$ & $4.8992 \mathrm{e}-6$ & 3.98 & $4.9353 e-6$ & 3.98 & $3.9280 \mathrm{e}-6$ & 3.97 \\
\hline
\end{tabular}

Table 3

Same as Table 1 , but in the direction of $\alpha=\frac{\pi}{2}$

\begin{tabular}{|c|c|c|c|c|c|c|}
\hline \multirow[t]{2}{*}{ Grid } & \multicolumn{2}{|l|}{$l_{1}$} & \multicolumn{2}{|l|}{$l_{2}$} & \multicolumn{2}{|l|}{$l_{\infty}$} \\
\hline & Error & Order & Error & Order & Error & Order \\
\hline $10 \times 10 \times 6$ & $1.6408 \mathrm{e}-2$ & - & $1.9294 \mathrm{e}-2$ & - & $1.9144 \mathrm{e}-2$ & - \\
\hline $20 \times 20 \times 6$ & $1.2546 \mathrm{e}-3$ & 3.71 & $1.4717 \mathrm{e}-3$ & 3.71 & $1.5198 \mathrm{e}-3$ & 3.65 \\
\hline $40 \times 40 \times 6$ & $8.3681 \mathrm{e}-5$ & 3.91 & $9.8352 \mathrm{e}-5$ & 3.90 & $1.0213 \mathrm{e}-4$ & 3.90 \\
\hline $80 \times 80 \times 6$ & $5.3194 \mathrm{e}-6$ & 3.98 & $6.2679 \mathrm{e}-6$ & 3.97 & $6.5277 \mathrm{e}-6$ & 3.98 \\
\hline
\end{tabular}




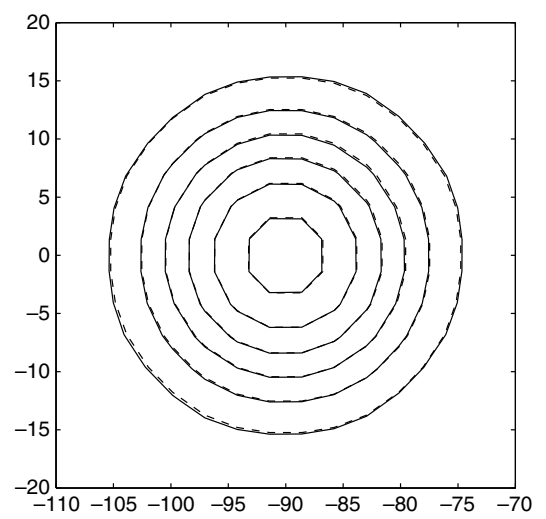

Fig. 7. Result of the cosine bell advection test on $32 \times 32 \times 6$ grid in the direction of $\alpha=\frac{\pi}{4}$. The contour lines plotted are from $100 \mathrm{~m}$ to $900 \mathrm{~m}$ by an interval of $160 \mathrm{~m}$. The solid line indicates the numerical result and the dashed line is the true solution.

Table 4

Errors of cosine bell advection test in different directions on $32 \times 32 \times 6$ grid

\begin{tabular}{|c|c|c|c|c|c|}
\hline Direction & $l_{1}$ & $l_{2}$ & $l_{\infty}$ & Maximum & Minimum \\
\hline$\alpha=0$ & $3.6248 \mathrm{e}-2$ & $2.3704 \mathrm{e}-2$ & $2.1207 \mathrm{e}-2$ & $-1.5726 \mathrm{e}-3$ & $-2.1207 \mathrm{e}-2$ \\
\hline$\alpha=\frac{\pi}{4}$ & $3.2008 \mathrm{e}-2$ & $1.9274 \mathrm{e}-2$ & $1.4880 \mathrm{e}-2$ & $-2.4309 \mathrm{e}-3$ & $-1.2957 \mathrm{e}-2$ \\
\hline$\alpha=\frac{4}{2}$ & $3.6310 \mathrm{e}-2$ & $2.3721 \mathrm{e}-2$ & $2.1215 \mathrm{e}-2$ & $-1.5548 \mathrm{e}-3$ & $-2.1215 \mathrm{e}-2$ \\
\hline
\end{tabular}

cubic grid (see [18], Fig. 4) with the similar resolution, our scheme produces much smaller errors when using the same CFL number. A flux-form semi-Lagrangian transport scheme based on PPM interpolation is proposed in [14] on the LAT/LON grid. Compared with the results by using FFSL-3 scheme (see [14], Table 6 ) on the $256 \times 128$ grid, a resolution doubly finer than that we used in this test, our numerical results look quite competitive in regard to all norms. Our numerical results are much better than those in [28] with the similar grid resolutions.

The time evolution of the normalized errors in different directions are given in Fig. 8. No obvious noises are generated by the patch boundaries and the numerical errors look almost independent of the flow direction. The present model guarantees the exact conservation of the mass. Shown in Fig. 8, the fluctuation of the total mass error is within the round-off limit.

It should be noted that we did not use any limiting projection to enforce the monotonicity of the numerical solutions in these tests though, a slope limiter proposed in the CSL3 reconstruction profile [40,11] has been proved quite effective in suppressing the spurious oscillations around the discontinuities, and can be adopted straightforwardly in the multi-dimensional reconstruction used in the present scheme.

\subsubsection{Deformational flow test}

The deformational flow test introduced in [22] is also presented here. It is considered to be more challenging than the solid rotation test. In rotated LAT/LON coordinates, the initial height field is specified by

$$
h=1-\tanh \left(\frac{\rho}{\gamma} \sin \lambda^{\prime}\right),
$$

where $\rho=\rho_{0} \cos \theta^{\prime}$ is the radius of the vortex. We specified $\rho_{0}=3$ and $\gamma=5$ as suggested in [24]. The flow field includes two opposite vortexes located at the two poles of the rotated coordinates. The angular velocity in the rotated LAT/LON coordinates is

$$
\omega= \begin{cases}\frac{3 \sqrt{3} \sinh ^{2} \rho \tanh \rho}{2 \rho} & \text { if } \rho \neq 0, \\ 0 & \text { if } \rho=0 .\end{cases}
$$



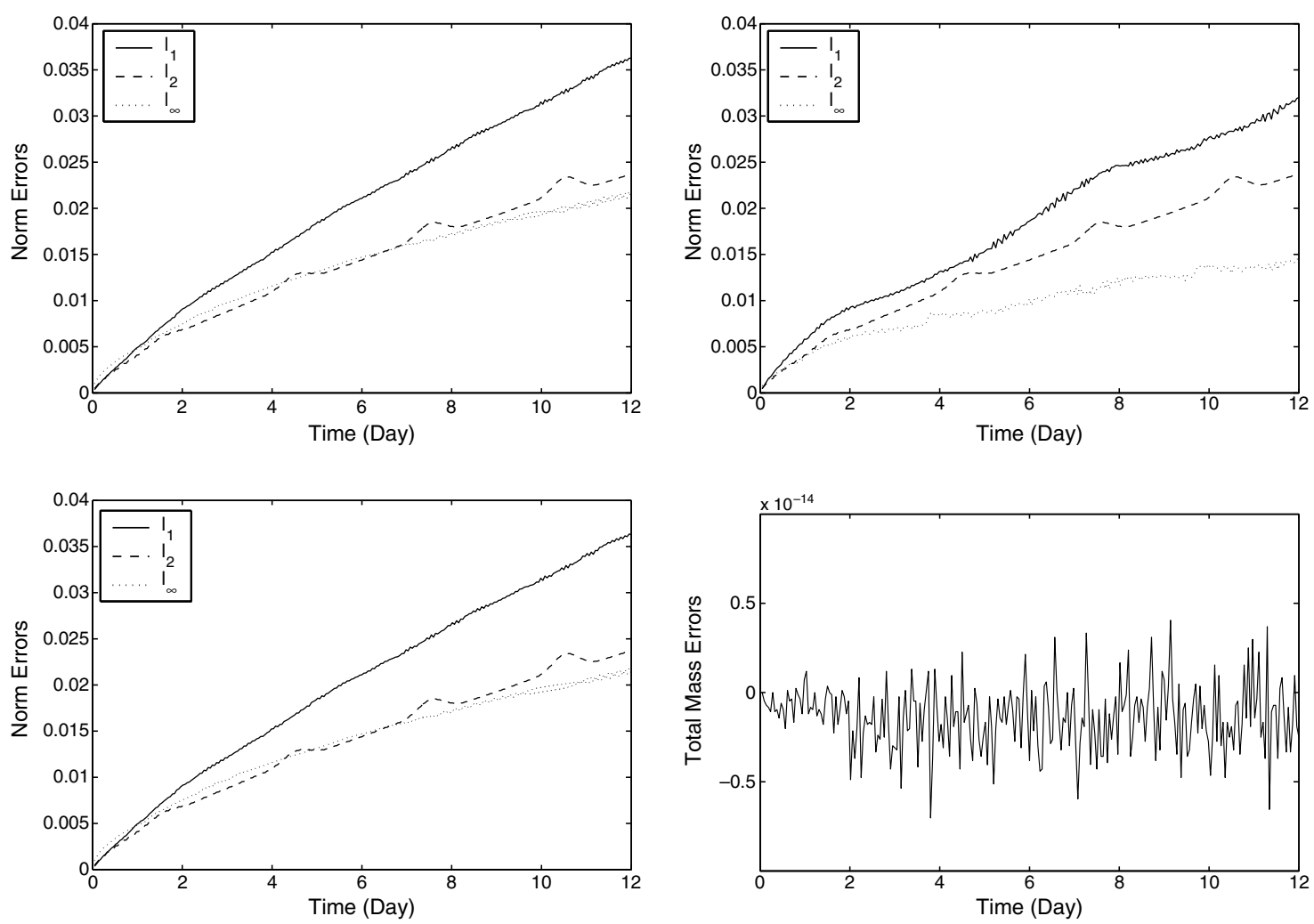

Fig. 8. The evolution of the normalized errors of the cosine bell advection on $32 \times 32 \times 6$ grid in the direction of $\alpha=0$ (top-left), $\alpha=\frac{\pi}{4}$ (top-right), $\alpha=\frac{\pi}{2}$ (bottom-left) and the evolution of the total mass error (bottom-right).

The analytic solution to this problem is known as

$$
h=1-\tanh \left[\frac{\rho}{\gamma} \sin \left(\lambda^{\prime}-\omega t\right)\right] .
$$

A detailed description of this test can be found in [21].

The initial height field is given by the top-left panel in Fig. 9. The contour lines ranging between 0.5 and 1.5 are plotted from right to left. The origin of the rotated coordinates is located at $\left(-\frac{\pi}{4},-\frac{3 \pi}{10}\right)$, thus a vortex is centered at one of the vertices of the inscribed cube. The test is carried out on the unit sphere $(R=1)$ and the time step of $\Delta t=\frac{2 \pi}{256}$ is used on a $32 \times 32 \times 6$ grid. The numerical result after integration for 128 steps is illustrated in the bottom-left panel of Fig. 9. The true solution at the same time is given in top-right panel for comparison. No visible difference can be found between the numerical result and the true solution. The absolute error (numerical result minus analytic field) is given in the bottom-right panel. The normalized errors are $l_{1}=1.2286 \times 10^{-5}, l_{2}=5.0733 \times 10^{-5}$ and $l_{3}=4.3632 \times 10^{-4}$ on $32 \times 32 \times 6$ grid.

\subsection{Shallow water equation tests}

The shallow water flows in spherical geometry are solved in the following numerical tests.

\subsubsection{Steady state geostrophic flow}

In this test, the initial height field is defined as

$$
g h=g h_{0}-\left(R \Omega u_{0}+\frac{u_{0}^{2}}{2}\right)(-\cos \lambda \cos \theta \sin \alpha+\sin \theta \cos \alpha)^{2},
$$



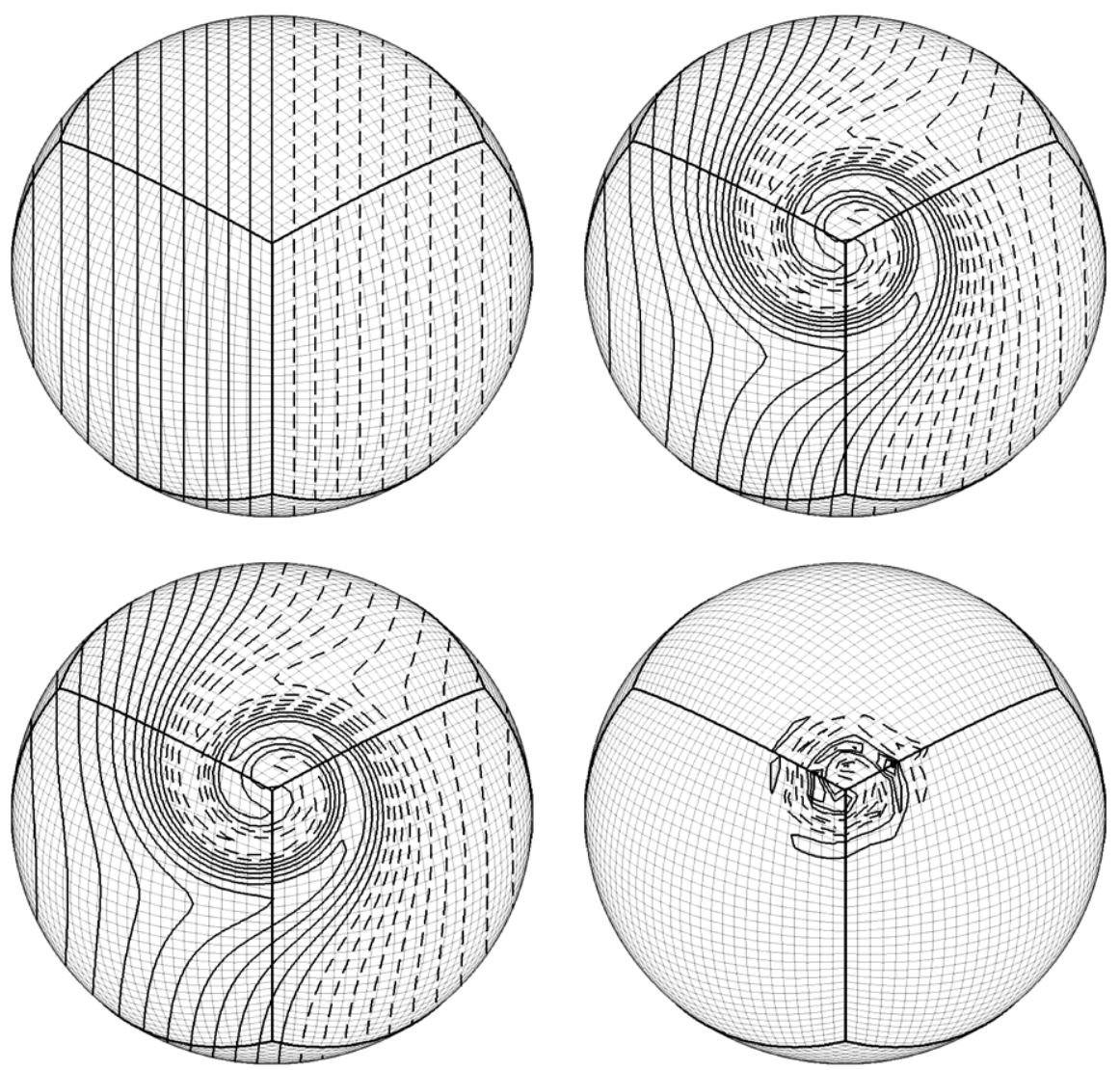

Fig. 9. Results of deformational flow on $32 \times 32 \times 6$ grid. Displayed are the initial height field (top-left), the analytic solution (top-right), the numerical result (bottom-left) and absolute error (bottom-right). The contour lines are from $0.5 \mathrm{~m}$ to $0.95 \mathrm{~m}$ by dashed lines and $1 \mathrm{~m}$ to $1.5 \mathrm{~m}$ by solid lines with an interval of $0.05 \mathrm{~m}$ for flow field, errors are plotted from $-8 \times 10^{-4} \mathrm{~m}$ to $-2 \times 10^{-4} \mathrm{~m}$ by dashed lines and $2 \times 10^{-4} \mathrm{~m}$ to $8 \times 10^{-4} \mathrm{~m}$ by solid lines with an interval of $2 \times 10^{-4} \mathrm{~m}$.

where $g h_{0}=2.94 \times 10^{4}$ and $u_{0}=2 \pi R /(12$ days $)$ and $\alpha$ is the angle between the rotation axis and polar axis of the Earth.

The divergence-free initial velocity field, same as previous advection test, is specified by (51). The Coriolis parameter is calculated as

$$
f=2 \Omega(-\cos \lambda \cos \theta \sin \alpha+\sin \theta \cos \alpha) .
$$

The balanced geostrophic relation holds in the initial distribution. So, both the height and flow fields should remain unchanging during the simulation. Again, using different values of the parameter $\alpha$, we tested the model with zonal flows of different orientations. The numerical results of height and wind fields after 5 model days for the flow in the direction of $\alpha=\frac{\pi}{4}$ are given in top panel of Fig. 10, and the absolute error of height field is given in bottom panel. We examined the convergence rate on three gradually refined grids with resolutions of $16 \times 16 \times 6,32 \times 32 \times 6$ and $64 \times 64 \times 6$. The normalized errors and convergence rates of the height field after 5 model days are given in Table 5 for northeast flow. According with the observation in the advection case, the 4 th-order convergence rate is obtained. We also checked the convergence rate with flows in the directions of $\alpha=0$ and $\alpha=\frac{\pi}{2}$, the results give the same convergence rate of 4 th-order.

A steady state nonlinear zonal flow test with compact support was also suggested in [36]. The flow field is similar to the above one except that the geostrophically balanced flow is limited to a belt region. The initial flow field is given analytically, but the initial height field should be obtained by numerical integration. The detailed description of this test can be found in [36]. We have run test in two flow directions, i.e. $\alpha=0$ and $\alpha=\frac{\pi}{3}$. The numerical result on $32 \times 32 \times 6$ grid after 5 days in the direction of $\alpha=\frac{\pi}{3}$ is shown in Fig. 11 . The normalized errors of flows in both directions are shown in Table 6. 

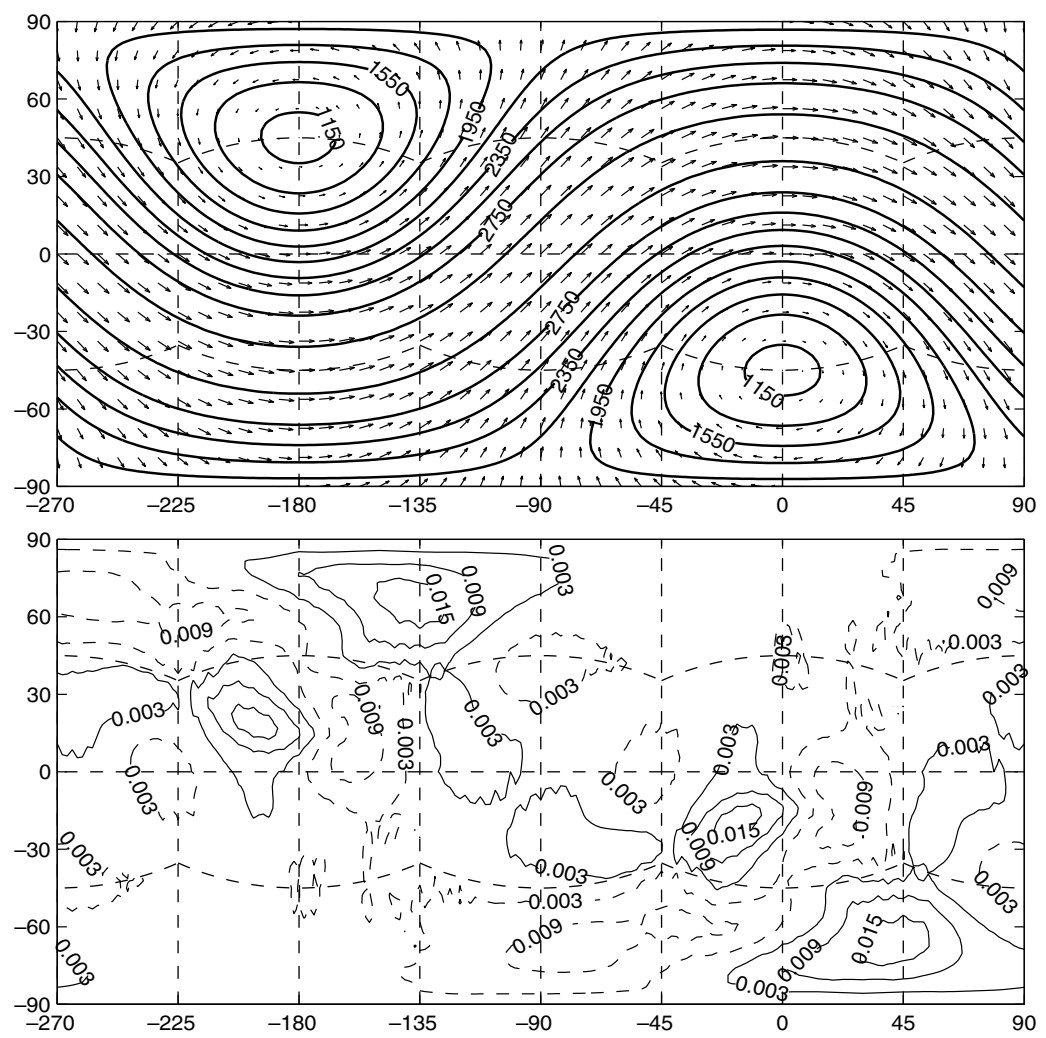

Fig. 10. Numerical result of steady state geostrophic flow in the direction of $\alpha=\frac{\pi}{4}$ on $32 \times 32 \times 6$ grid. The height field and wind field at day 5 (top),contour lines are plotted from $1150 \mathrm{~m}$ to $2950 \mathrm{~m}$ with an interval of $200 \mathrm{~m}$. The absolute error of height field (bottom), contour lines are plotted from $-0.015 \mathrm{~m}$ to $-0.003 \mathrm{~m}$ by dashed lines and $0.003 \mathrm{~m}$ to $0.015 \mathrm{~m}$ by solid lines with an interval of $0.003 \mathrm{~m}$.

Table 5

Errors and convergence rate of steady state flow in the direction of $\alpha=\frac{\pi}{4}$

\begin{tabular}{|c|c|c|c|c|c|c|}
\hline \multirow[t]{2}{*}{ Grid } & \multicolumn{2}{|l|}{$l_{1}$} & \multicolumn{2}{|l|}{$l_{2}$} & \multicolumn{2}{|l|}{$l_{\infty}$} \\
\hline & Error & Order & Error & Order & Error & Order \\
\hline $16 \times 16 \times 6$ & $2.4438 \mathrm{e}-5$ & - & $3.2519 \mathrm{e}-5$ & - & $9.7940 \mathrm{e}-5$ & - \\
\hline $32 \times 32 \times 6$ & $1.6008 \mathrm{e}-6$ & 3.93 & $2.1202 \mathrm{e}-6$ & 3.94 & $5.8647 \mathrm{e}-6$ & 4.06 \\
\hline $64 \times 64 \times 6$ & $1.0199 \mathrm{e}-7$ & 3.97 & $1.3462 \mathrm{e}-7$ & 3.98 & $3.3126 \mathrm{e}-7$ & 4.15 \\
\hline
\end{tabular}

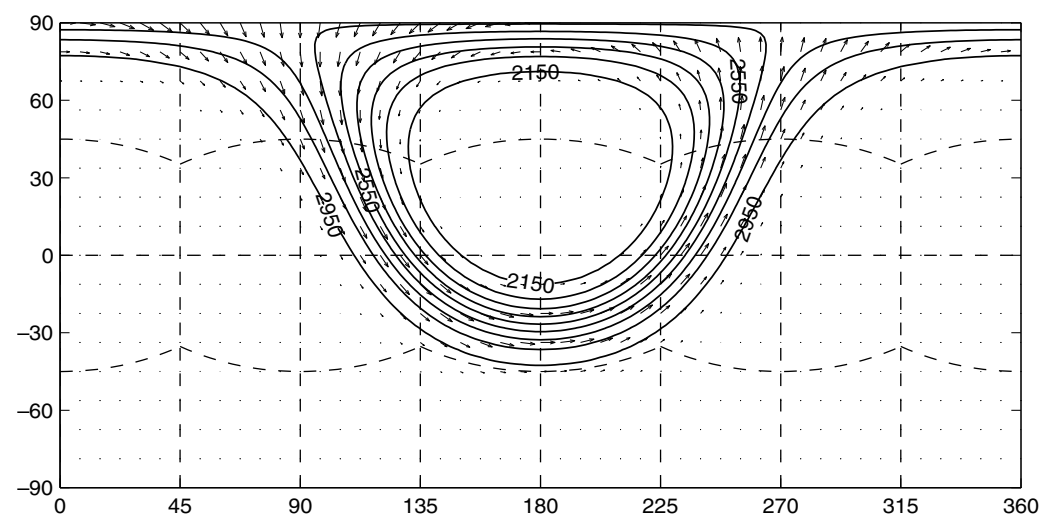

Fig. 11. Numerical result of steady state geostrophic flow with compact support in the direction of $\alpha=\frac{\pi}{3}$ on $32 \times 32 \times 6$ grid. Contour lines are plotted from $2150 \mathrm{~m}$ to $2950 \mathrm{~m}$ with an interval of $100 \mathrm{~m}$. 
Table 6

Errors of steady state flow with compact support in different directions on $32 \times 32 \times 6$ grid

\begin{tabular}{llll}
\hline Direction & $l_{1}$ & $l_{2}$ & $l_{\infty}$ \\
\hline$\alpha=0$ & $7.0866 \mathrm{e}-6$ & $1.2036 \mathrm{e}-5$ & $7.3831 \mathrm{e}-5$ \\
$\alpha=\frac{\pi}{3}$ & $7.9558 \mathrm{e}-6$ & $1.5021 \mathrm{e}-5$ & $7.7302 \mathrm{e}-5$ \\
\hline
\end{tabular}

\subsubsection{Zonal flow over an isolated mountain}

This test is used to check the performance of the numerical model in solving a problem including a topographic term. A zonal flow is the same as in steady state flow test in Section 4.2.1 except the parameters are modified as $h_{0}=5960 \mathrm{~m}$ and $u_{0}=20 \mathrm{~m} / \mathrm{s}$. A bottom mountain is centered at $\left(\lambda_{c}, \theta_{c}\right)=\left(\frac{3 \pi}{2}, \frac{\pi}{6}\right)$, and the height of the mountain is analytically given as
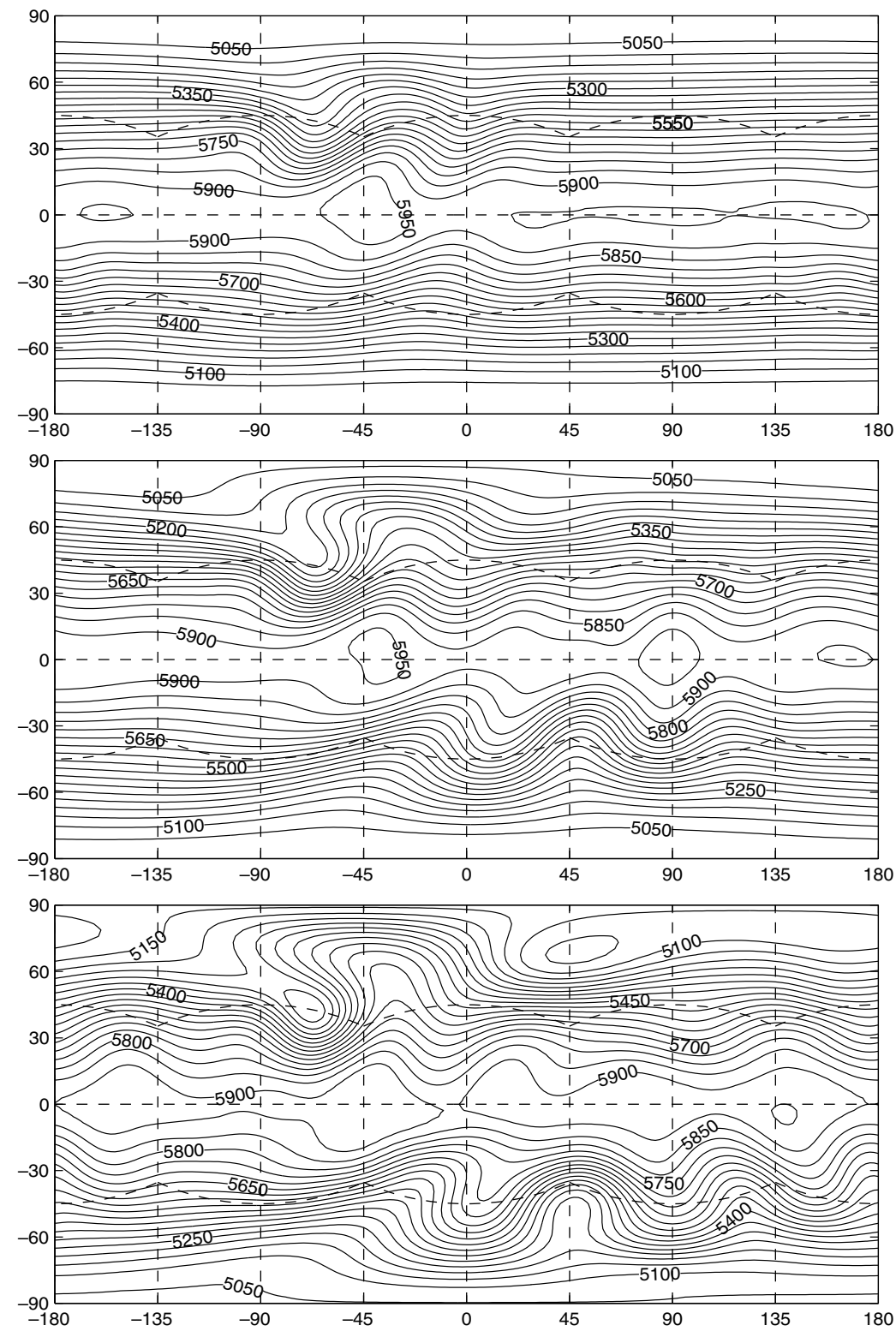

Fig. 12. Results of the zonal flow over an isolated mountain on $32 \times 32 \times 6$ grid. Shown are the total height at day 5 (top), day 10 (middle) and day 15 (bottom). Contour lines are plotted from $5050 \mathrm{~m}$ to $5950 \mathrm{~m}$ with an interval of $50 \mathrm{~m}$. 


$$
h_{\mathrm{s}}=h_{s 0}\left(1-\frac{r}{r_{0}}\right) \text {, }
$$

where $h_{s 0}=2000 \mathrm{~m}, r_{0}=\frac{\pi}{9}$ and $r=\min \left[r_{0}, \sqrt{\left(\lambda-\lambda_{c}\right)^{2}+\left(\theta-\theta_{c}\right)^{2}}\right]$.

The analytic solution to this test is not available. We compared our results with a reference solution from a spectral model with high resolution. The numerical results for height field by the present model at day 5,10 and 15 are given in Fig. 12. The resolution used is $32 \times 32 \times 6$. The numerical result calculated by a spectral method on the T213 grid (see [13], Fig. 5.1) is taken as the reference solution for validating our numerical model. From the comparison, we found that our numerical model accurately reproduces the height field. Meanwhile, the oscillations occurring at the boundary of the mountain are effectively eliminated by using the well-balanced formulation for the topographic source term, as described at the end of Section 3.1. The evolution of normalized errors of total mass, total energy and potential enstrophy is given in Fig. 13. Our scheme conserves the total mass to the machine round-off precision, the errors of the total energy and potential enstrophy are competitive to the results by the spectral transform method on the T63 grid (see [12], Fig. 5.5). Compared with the results by TVD wave propagation method on $128 \times 128 \times 6$ grid (see [28], Fig. 9), the total energy error is one order smaller and the error of potential enstrophy has the similar magnitude.

\subsubsection{Rossby-Haurwitz wave}

A 4-wave Rossby-Haurwitz wave is adopted as test 6 in Williamson's standard test set. The divergence-free flow field is given by the stream function as

$$
\psi=-R^{2} \omega \sin \theta+R^{2} K \cos ^{r} \theta \sin \theta \cos r \lambda,
$$

and the initial height field is specified as

$$
g h=g h_{0}+R^{2} A+R^{2} B \cos r \lambda+R^{2} C \cos 2 r \lambda,
$$

where $\omega, r$ and $K$ are the constants, $\omega=K=7.848 \times 10^{-6} \mathrm{~s}^{-1}$ and $r=4 . A, B, C$ are the functions of the latitude. The detailed expressions can be found in [36]. Some comments on the properties of Rossby-Haurwitz wave and its numerical solutions from some global models can be found in [33]. Due to its intrinsic dynamic instability, this might not be a proper example for testing the long-term performance of a numerical model. Nevertheless, the Rossby-Haurwitz wave still provides a good test bed for global middle-term simulations. The numerical results up to 14 days calculated by the spectral method on the T213 grid (see [13], Fig. 5.5) are widely accepted as the reference solutions that reasonably reflect the dynamic behavior of the system.

We plot our numerical results of the height fields after 7 and 14 days on $48 \times 48 \times 6$ grid in Fig. 14 together with the initial height field. It is evident that our numerical results agree well with the reference solutions [13]. The evolution of normalized errors of total energy and potential enstrophy on $32 \times 32 \times 6$ grid is given in
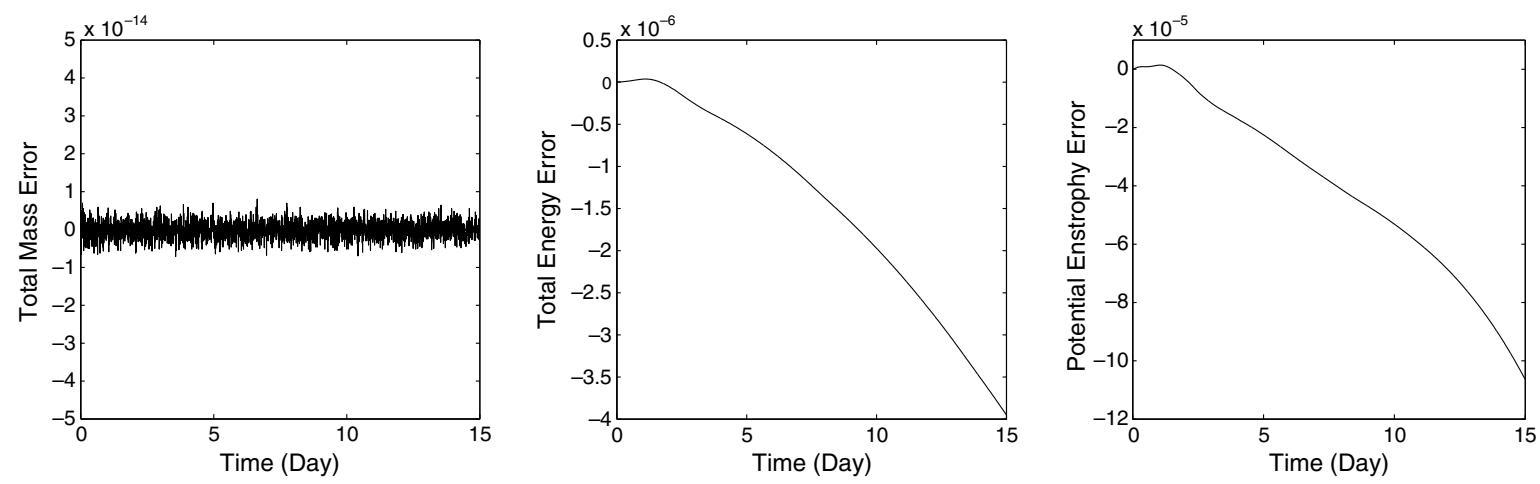

Fig. 13. Evolution of the normalized conservation errors of total mass (left), total energy (middle) and potential enstrophy (right) on $32 \times 32 \times 6$ grid for the zonal flow over an isolated mountain test. 

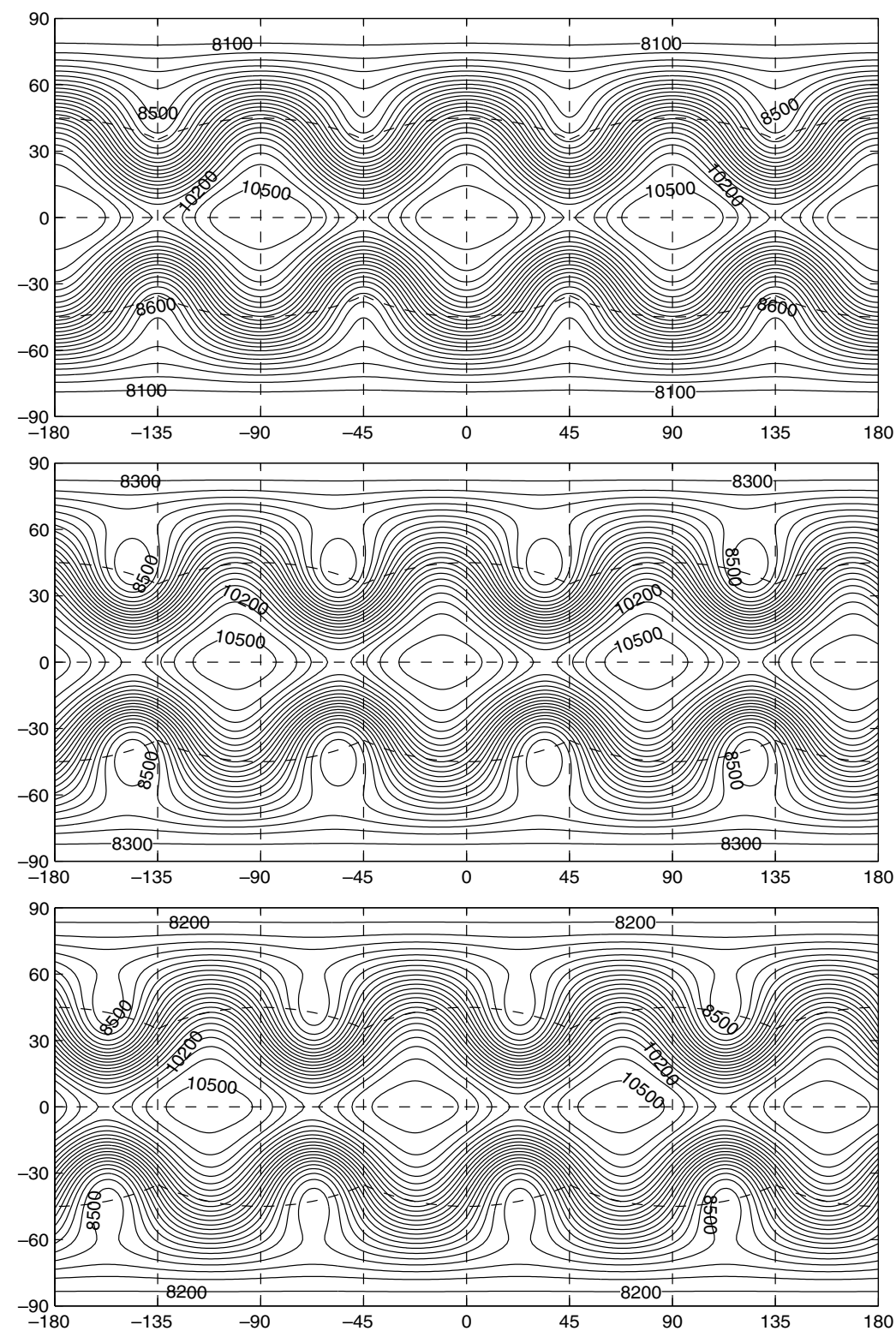

Fig. 14. Numerical results of Rossby-Haurwitz wave on $48 \times 48 \times 6$ grid. Displayed are the initial height (top), the height fields at day 7 (middle) and day 14 (bottom). Contour lines are plotted from 8100 to $105,00 \mathrm{~m}$ with an interval of $100 \mathrm{~m}$.

Fig. 15. Compared with the results by a flux-form semi-Lagrangian model on a LAT/LON grid of same resolution (see [15], Fig. 7), the error of the total energy is one order smaller and the error of potential enstrophy is only half in the present results. Even compared with the results using the spectral transform methods on T63 grid (see [12], Fig. 6.7), the total energy error of our model is smaller and the potential enstrophy error looks quite competitive. Moreover, the present model exactly guarantees both local and global conservation of the total mass.

\subsubsection{Barotropic instability test}

The barotropic instability test introduced in [6] is a useful complement of Williamson's standard test set, particularly for a numerical model built on the cubed-sphere grid. 

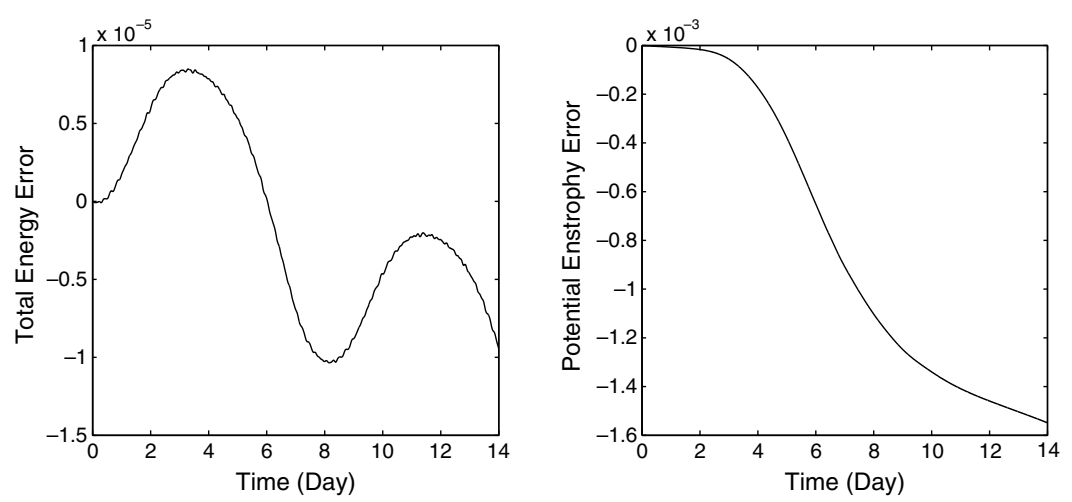

Fig. 15. Evolution of the normalized conservation errors of total energy (left) and potential enstrophy (right) on $32 \times 32 \times 6$ grid for Rossby-Haurwitz wave test, results are competitive to the results by reference spectral method on T63 grid.

The zonal flow field is given by

$$
u_{\lambda}(\theta)= \begin{cases}0 & \text { if } \theta \leqslant \theta_{0}, \\ \frac{u_{\max }}{e_{n}} \exp \left[\frac{1}{\left(\theta-\theta_{0}\right)\left(\theta-\theta_{1}\right)}\right] & \text { if } \theta_{0}<\theta<\theta_{1}, \\ 0 & \text { if } \theta \geqslant \theta_{1},\end{cases}
$$

where $u_{\max }=80 \mathrm{~m} / \mathrm{s}, \theta_{0}=\frac{\pi}{7}, \theta_{1}=\frac{\pi}{2}-\theta_{0}$ and $e_{n}=\exp \left[-4 /\left(\theta_{1}-\theta_{0}\right)^{2}\right]$.

The basic balanced height field can be obtained by integrating the following balance equation

$$
g h(\theta)=g h_{0}-\int_{-\frac{\pi}{2}}^{\theta} R u_{\lambda}\left(\theta^{\prime}\right)\left[f+\frac{\tan \left(\theta^{\prime}\right)}{R} u_{\lambda}\left(\theta^{\prime}\right)\right] \mathrm{d} \theta^{\prime},
$$

where $h_{0}=10000 \mathrm{~m}$.

An initial perturbation of height field is added to the balanced flow to initiate the instability as

$$
h^{\prime}=\hat{h} \cos (\theta) \exp \left[-\left(\frac{\lambda}{\alpha}\right)^{2}-\left(\frac{\theta_{2}-\theta}{\beta}\right)^{2}\right],
$$

where $\hat{h}=120 \mathrm{~m}, \alpha=\frac{1}{3}, \beta=\frac{1}{15}$ and $\theta_{2}=\frac{\pi}{4}$.

This test is very challenging for the numerical model constructed on the cubed-sphere. The height field changes with a much larger gradient near the patch boundaries than Williamson's test 2. Meanwhile, the initial instability is located on the boundary between patch one and patch five. So, the numerical errors generated by the discontinuous coordinates along the boundaries easily pollute the numerical results. We run the multimoment model on the gradually refined grids of $32 \times 32 \times 6,64 \times 64 \times 6,96 \times 96 \times 6$ and $128 \times 128 \times 6$. The height fields after 6 days are shown by contour plots in Fig. 16.

As expected, the numerical result on the $32 \times 32 \times 6$ grid is significantly affected by the boundary errors. The 4-wave phenomena in relative vorticity field is observed. With increasing the resolution, the numerical results converge to the reference solution (see [6], Fig. 4) on the finer grids. Though the result on the $64 \times 64 \times 6$ grid still influenced by the 4 -wave errors, the results on the last two grids look very similar and accord with the reference solution. Furthermore, no explicit diffusion is added during the computation in our test, and the small scales in the numerical solution can be captured better by our model.

\section{Summary}

A numerical model for global shallow water flows by the CIP/multi-moment finite volume method on the cubed-sphere grid has been developed. With the application of the cubed-sphere grid to the spherical geometry, a structured computational grid with uniform mesh spacing and free of polar singularity is obtained on the whole globe. A 4th-order numerical model is constructed by making use of multi-moment concept that leads to a very compact computational stencil. The multi-moment formulation also makes the numerical treat- 

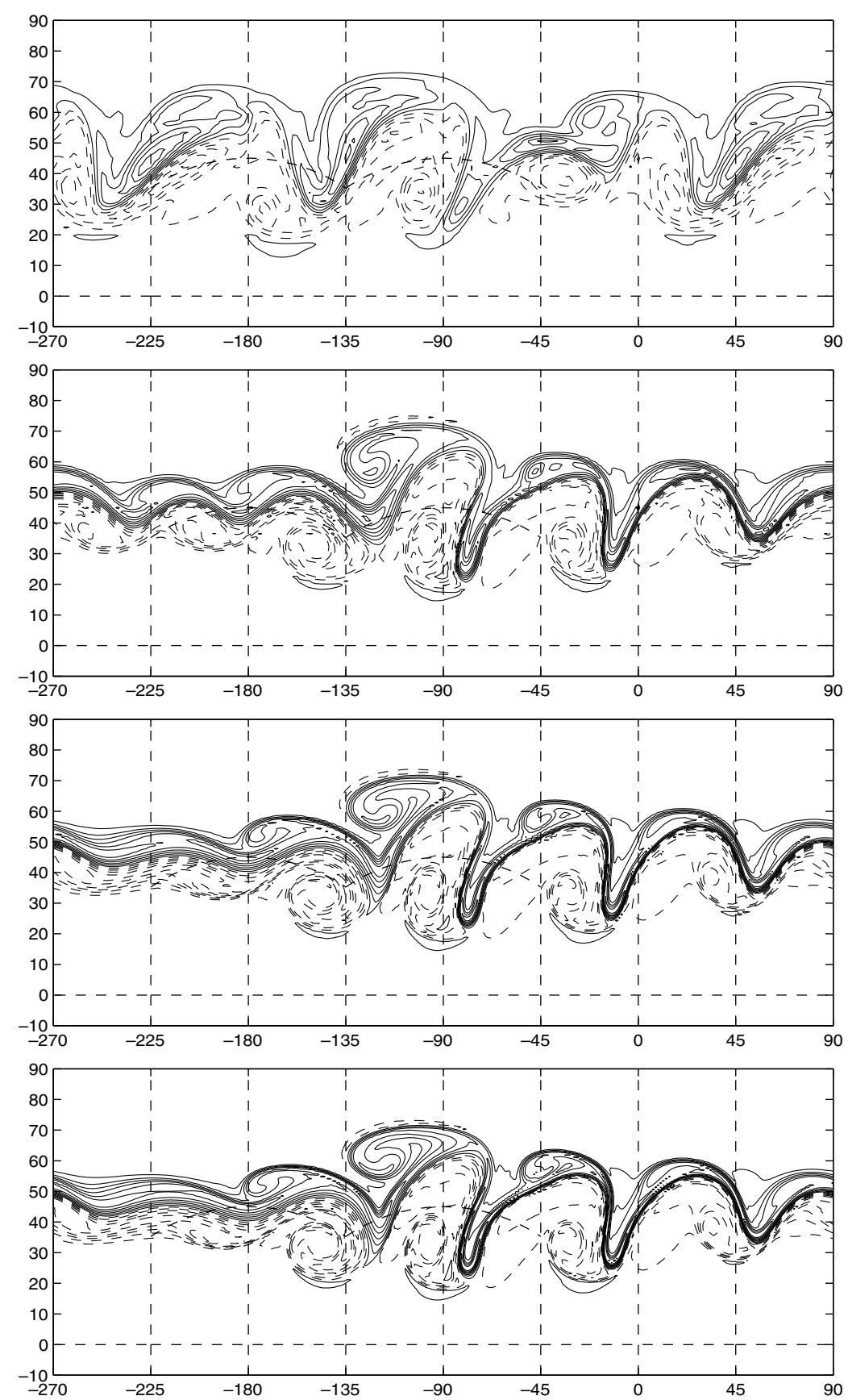

Fig. 16. The vorticity fields of the barotropic instability test at day 6 by multi-moment model on gradually refined resolutions, $32 \times 32 \times 6$ grid ( 1 st panel from top), $64 \times 64 \times 6$ grid ( 2 nd panel), $96 \times 96 \times 6$ grid ( 3 rd panel) and $128 \times 128 \times 6$ grid (4th panel). Contour lines are from $-1.1 \times 10^{-4} \mathrm{~s}^{-1}$ to $-0.1 \times 10^{-4} \mathrm{~s}^{-1}$ by dashed lines and from $0.1 \times 10^{-4} \mathrm{~s}^{-1}$ to $1.5 \times 10^{-4} \mathrm{~s}^{-1}$ by solid lines with an interval of $0.2 \times 10^{-4} \mathrm{~s}^{-1}$.

ments across the patch boundary much easier. With one of the moment, i.e. the volume-integrated average updated by a finite volume formulation of flux form, the rigorous numerical conservation is achieved.

The numerical model has been extensively verified by numerical experiments including the widely used benchmark problems. The numerical results reveal that the model is competitive to the most existing ones regarding robustness, accuracy and computational efficiency. We can expect the present model as a promising numerical framework for global geophysical fluid dynamics. 


\section{Appendix A}

Given the VIA and PVs, the constrained conditions for (30) of control volume $\mathcal{C}_{i j}$ are imposed as the following set of equations

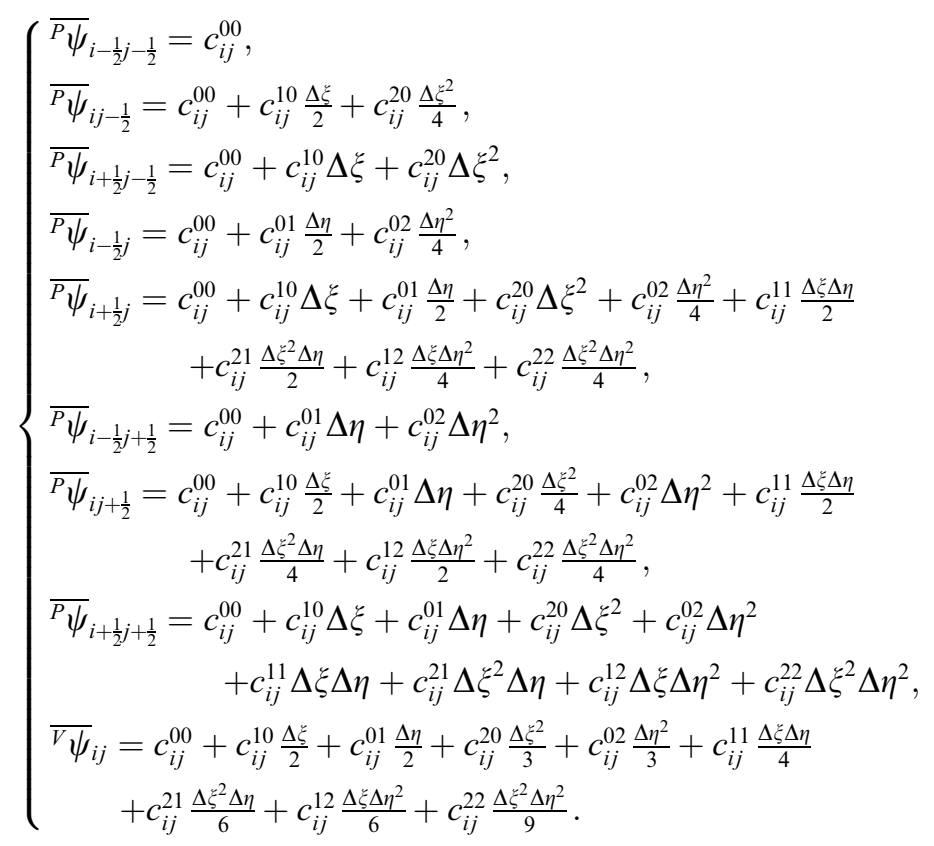

Solving (64), one gets the coefficients of the interpolation function as

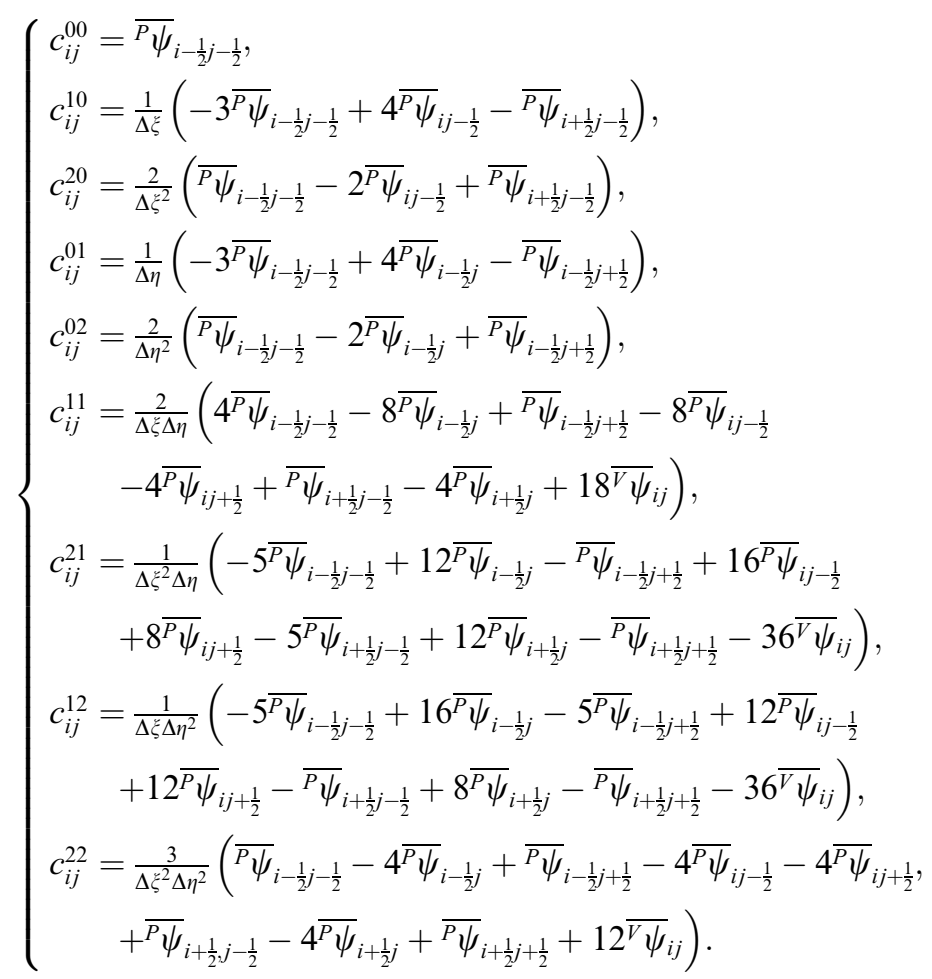




\section{References}

[1] M. Abramowitz, I.A. Stegun, Handbook of mathematical functions with formulas, graphs, and mathematical tables, ninth dover printing, tenth gpo printing ed., Dover, New York, 1964.

[2] A. Adcroft, J.-M. Campin, C. Hill, J. Marshall, Implementation of an atmosphere-ocean general circulation model on expanded spherical cube, Mon. Weather Rev. 132 (12) (2004) 2845-2863.

[3] A. Bermudez, M.E. Vazquez, Upwind methods for hyperbolic conservation laws with source terms, Comput. Fluids 23 (8) (1994) 1049-1071.

[4] B. Cockburn, C.W. Shu, The Runge-Kutta discontinuous Galerkin method for conservation laws V: multidimensional systems, J. Comput. Phys. 141 (2) (1998) 199-224.

[5] J. Côté, S. Gravel, A. Méthot, A. Patoine, M. Roch, A. Staniforth, The operational CMC-MRB global environmental multiscale (GEM) model. part I: design considerations and formulation, Mon. Weather Rev. 126 (6) (1998) 1373-1395.

[6] J. Galewsky, R.K. Scott, L.M. Polvani, An initial-value problem for testing numerical models of the global shallow-water equations, Tellus 56A (2004) 429-440.

[7] F. Giraldo, T.E. Rosmond, A scalable spectral element Eulerian atmospheric model (SEE-AM) for NWP: dynamical core tests, Mon. Weather Rev. 132 (1) (2004) 133-153.

[8] R. Heikes, D.A. Randall, Numerical integration of the shallow-water equations on a twisted icosahedral grid. part I: basic design and results of tests, Mon. Weather Rev. 123 (6) (1995) 1862-1880.

[9] W. Hundsdorfer, E. Spee, An efficient horizontal advection scheme for the modeling of global transport of constituents, Mon. Weather Rev. 123 (12) (1995) 3554-3564.

[10] S. Ii, M. Shimuta, F. Xiao, A 4th-order and single-cell-based advection scheme on unstructured grids using multi-moments, Comput. Phys. Commun. 173 (1-2) (2005) 17-33.

[11] S. Ii, F. Xiao, CIP/multi-moment finite volume method for Euler equations: a semi-Lagrangian characteristic formulation, J. Comput. Phys. 222 (2) (2007) 849-871.

[12] R. Jakob, J.J. Hack, D.L. Willamson, Solutions to the shallow water test set using the spectral transform method, Tech. Rep. NCAR/ TN-388 + STR, National Center for Atmospheric Research, 1993.

[13] R. Jakob-Chien, J.J. Hack, D.L. Williamson, Spectral transform solutions to the shallow water test set, J. Comput. Phys. 119 (1) (1995) 164-187.

[14] S.J. Lin, R.B. Rood, Multidimensional flux-form semi-Lagrangian transport schemes, Mon. Weather Rev. 124 (9) (1996) $2046-2070$.

[15] S.J. Lin, R.B. Rood, An explicit flux-form semi-Lagrangian shallow water model on the sphere, Quart. J. Roy. Meteorol. Soc. 123 (544) (1997) 2477-2498.

[16] D. Majewski, D. Liermann, P. Prohl, B. Ritter, M. Buchhold, T. Hanisch, G. Paul, W. Wergen, J. Baumgardner, The operational global icosahedralhexagonal gridpoint model GME: description and high-resolution tests, Mon. Weather. Rev. 130 (2) (2002) 319 338.

[17] A. McDonald, J.R. Bates, Semi-Lagrangian integration of a gridpoint shallow water model on the sphere, Mon. Weather. Rev. 117 (1) (1989) $130-137$.

[18] J.L. McGregor, Semi-Lagrangian advection on conformal-cubic grids, Mon. Weather. Rev. 124 (6) (1996) $1311-1322$.

[19] J.L. McGregor, Semi-Lagrangian advection on a cubic gnomonic projection of the sphere, Atmos.-Ocean. Memor. Vol. (1997) 153169.

[20] J.L. McGregor, C-CAM: geometric aspects and dynamical formulation, Tech. Rep. CSIRO Atmospheric Research Tech. Paper No. 70, CSIRO, 2005.

[21] R.D. Nair, J. Côté, A. Staniforth, Cascade interpolation for semi- Lagrangian advection over the sphere, Quart. J. Roy. Meteorol. Soc. 125 (556) (1999) 1445-1468.

[22] R.D. Nair, B. Machenhauer, The mass-conservative cell-integrated semilagrangian advection scheme on the sphere, Mon. Weather. Rev. 130 (3) (2002) 649-667.

[23] R.D. Nair, S.J. Thomas, R.D. Loft, A discontinuous Galerkin global shallow water model, Mon. Weather. Rev. 133 (4) (2005) 876887.

[24] R.D. Nair, S.J. Thomas, R.D. Loft, A discontinuous Galerkin transport scheme on the cubed sphere, Mon. Weather. Rev. 133 (4) (2005) 827-841.

[25] M. Rancic, R.J. Purser, F. Mesinger, A global shallow-water model using an expanded spherical cube: gnomonic versus conformal coordinates, Quart. J. Roy. Meteorol. Soc. 122 (532) (1996) 959-982.

[26] P.J. Rasch, Conservative shape-preserving two-dimensional transport on a spherical reduced grid, Mon. Weather. Rev. 122 (6) (1994) 1337-1350.

[27] C. Ronchi, R. Iacono, P.S. Paolucci, The "Cubed Sphere": a new method for the solution of partial differential equations in spherical geometry, J. Comput. Phys. 124 (1) (1996) 93-114.

[28] J.A. Rossmanith, A wave propagation method for hyperbolic systems on the sphere, J. Comput. Phys. 213 (2) (2006) 629-658.

[29] R. Sadourny, Conservative finite-difference approximations of the primitive equations on quasi-uniform spherical grids, Mon. Weather. Rev. 100 (2) (1972) 136-144.

[30] R. Sadourny, A. Arakawa, Y. Mintz, Integration of the nondivergent barotropic vorticity equation with an icosahedral-hexagonal grid for the sphere, Mon. Weather. Rev. 96 (6) (1968) 351-356.

[31] G.R. Stuhne, W.R. Peltier, New icosahedral grid-point discretizations of the shallow water equations on the sphere, J. Comput. Phys. 148 (1) (1999) 23-58. 
[32] S.J. Thomas, R.D. Loft, Semi-implicit spectral element atmospheric model, J. Sci. Comput. 17 (1-4) (2002) 339-350.

[33] J. Thuburn, Y. Li, Numericial simulations of Rossby-Haurwitz waves, Tellus 52A (2) (2000) 181-189.

[34] H. Tomita, M. Tsugawa, M. Satoh, K. Goto, Shallow water model on a modified icosahedral geodesic grid by using spring dynamics, J. Comput. Phys. 174 (2) (2001) 579-613.

[35] D.L. Williamson, Integration of the barotropic vorticity equation on a spherical geodesic grid, Tellus 20 (4) (1968) $642-653$.

[36] D.L. Williamson, J.B. Drake, J.J. Hack, R. Jakob, P.N. Swarztrauber, A standard test set for numerical approximations to the shallow water equations in spherical geometry, J. Comput. Phys. 102 (1) (1992) 211-224.

[37] F. Xiao, Unified formulation for compressible and incompressible flows by using multi-integrated moments I: one-dimensional inviscid compressible flow, J. Comput. Phys. 195 (2) (2004) 629-654.

[38] F. Xiao, R. Akoh, S. Ii, Unified formulation for compressible and incompressible flows by using multi-integrated moments II: multidimensional version for compressible and incompressible flows, J. Comput. Phys. 213 (1) (2006) 31-56.

[39] F. Xiao, X. Peng, X. Shen, A finite volume grid using multi-moments for geostrophic adjustment, Mon. Weather. Rev. 134 (1) (2006) 2515-2526.

[40] F. Xiao, T. Yabe, Completely conservative and oscillationless semi-Lagrangian schemes for advection transportation, J. Comput. Phys. 170 (2) (2001) 498-522.

[41] F. Xiao, T. Yabe, X. Peng, H. Kobayashi, Conservation and oscillationless transport schemes based on rational functions, J. Geophys. Res. 107 (D22) (2002) 642-653.

[42] Y. Xing, C.W. Shu, High order finite difference WENO schemes with the exact conservation property for the shallow water equations, J. Comput. Phys. 208 (1) (2005) 206-227.

[43] T. Yabe, R. Tanaka, T. Nakamura, F. Xiao, An exactly conservative semi-Lagrangian scheme (CIP-CSL) in one dimension, Mon. Weather. Rev. 129 (2) (2001) 332-344.

[44] M.P. Zhang, C.W. Shu, An analysis of and a comparison between the discontinuous Galerkin and the spectral finite volume methods, Comput. Fluids 34 (4-5) (2005) 581-592. 